Issued by Sandia National Laboratories, operated for the United States Department of Energy by Sandia Corporation.

NOTICE: This report was prepared as an account of work sponsored by an agency of the United States Government. Neither the United States Government, nor any agency thereof, nor any of their employees, nor any of their contractors, subcontractors, or their employees, make any warranty, express or implied, or assume any legal liability or responsibility for the accuracy, completeness, or usefulness of any information, apparatus, product, or process disclosed, or represent that its use would not infringe privately owned rights. Reference herein to any specific commercial product, process, or service by trade name, trademark, manufacturer, or otherwise, does not necessarily constitute or imply its endorsement, recommendation, or favoring by the United States Government, any agency thereof, or any of their contractors or subcontractors. The views and opinions expressed herein do not necessarily state or reflect those of the United States Government, any agency thereof, or any of their contractors.

Printed in the United States of America. This report has been reproduced directly from the best available copy.

Available to DOE and DOE contractors from

Office of Scientific and Technical Information

P.O. Box 62

Oak Ridge, TN 37831

Prices available from (703) 605-6000

Web site: http://www.ntis.gov/ordering.htm

Available to the public from

National Technical Information Service

U.S. Department of Commerce

5285 Port Royal Rd

Springfield, VA 22161

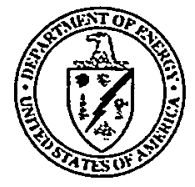




\section{DISCLAIMER}

Portions of this document may be illegible in electronic image products. Images are produced from the best available original document. 
SAND 99-3150

Unlimited Release

Printed January 2000

\title{
Forensic Imaging Tools for Law Enforcement
}

\author{
Colin Smithpeter, Ph.D., Dave Sandison, Ph.D., and Tim Vargo \\ Telemetry Technology Development \\ Sandia National Laboratories \\ Box 5800 \\ Albuquerque, NM 87185-0987
}

\begin{abstract}
Conventional methods of gathering forensic evidence at crime scenes are encumbered by difficulties that limit local law enforcement's efforts to apprehend offenders and bring them to justice. Working with a local law-enforcement agency, Sandia National Laboratories has developed a prototype multispectral imaging system that can speed up the investigative search task and provide additional and more accurate evidence. The system, called the Criminalistics Light-imaging Unit (CLU), has demonstrated the capabilities of locating fluorescing evidence at crime scenes under normal lighting conditions and of imaging other types of evidence, such as untreated fingerprints, by direct white-light reflectance. CLU employs state of the art technology that provides for viewing and recording of the entire search process on videotape. This report describes the work performed by Sandia to design, build, evaluate, and commercialize CLU.
\end{abstract}




\section{Acknowledgments}

The authors greatly appreciate the invaluable support of the Albuquerque Police Department (APD) Crime Lab for its contributions to this project. The APD Crime Lab, which received no funding for this work, took an interest in developing new technology and provided crucial input during the design process. Special thanks to Director Ann Talbot for allowing us access to her people, crime scenes, and expertise; to Detective J. D. Herrera for leading our interaction and spending so much time with us; to Detective Joe Foster for his enthusiasm and input; and to Catherine Dickey for preparing test samples and participating in the tests. We also appreciate the contributions of many other detectives and lab members who provided input and sample evidence.

The contributions of employees at Sandia National Laboratories are also much appreciated. Thanks to Program Manager Debra Spencer for her guidance, to Tim Duba for his hard work in assembling the instrument, and to Dave Sandison for originating the project. Thanks also to Rhonda Reinert for assembling, editing, and publishing this report.

This project would not have been possible without the support of the National Institute of Justice. Special thanks to David Boyd for showing interest and supporting the funding of this work; to Richard Rao for funding the development of CLU, assisting us in understanding law enforcement needs, and suggesting that we include a corporate partner; and to Lisa Forman for supporting development efforts and providing feedback to us throughout the project. 


\section{Contents}

Acronyms and Abbreviations................................................................................. 5

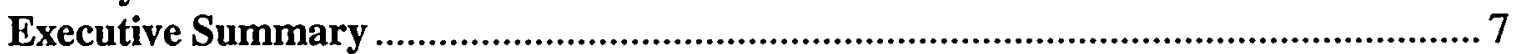

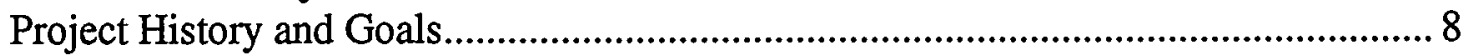

System Capabilities ................................................................................................. 8

Future System Development ..................................................................................... 9

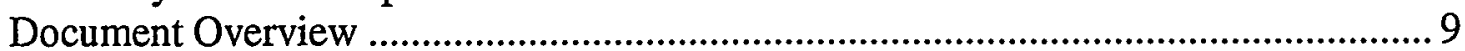

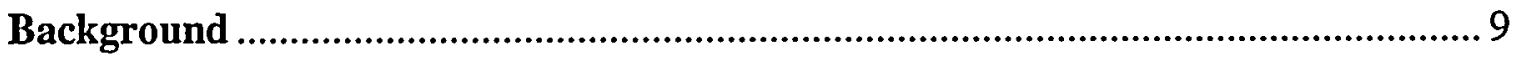

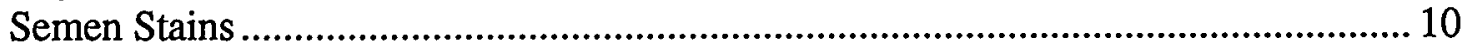

Blood Spatter Patterns and Trails ................................................................................ 12

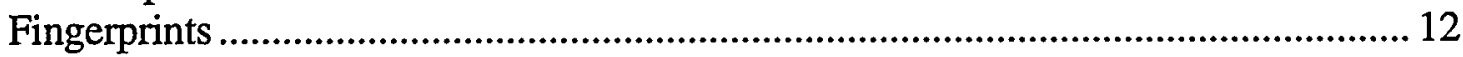

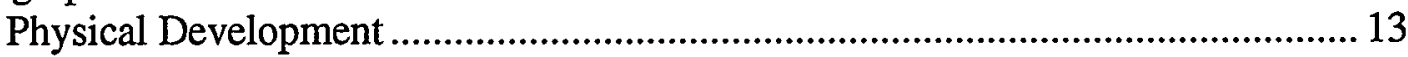

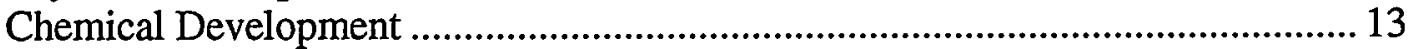

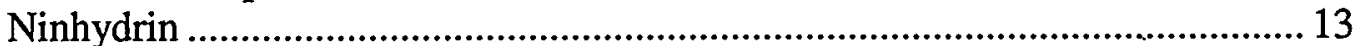

Cyanoacrylate Fuming.............................................................................. 14

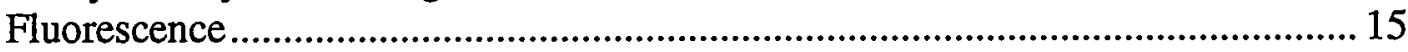

Investigations into Using Inherent Fluorescence .............................................. 15

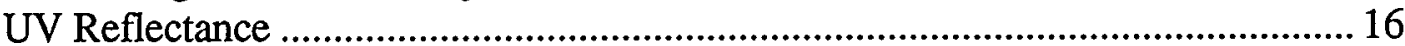

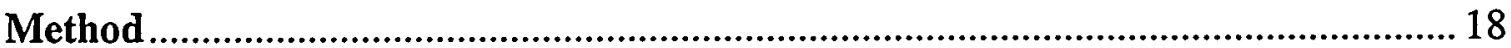

Technical Approach and Design Considerations ..................................................... 18

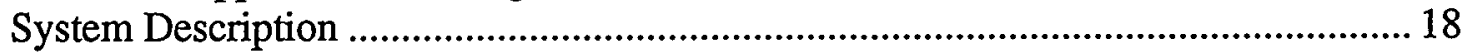

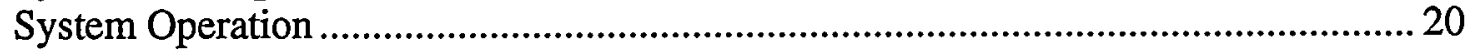

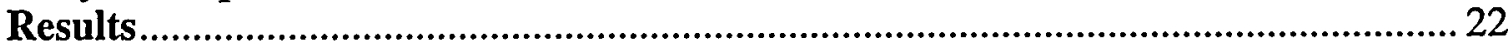

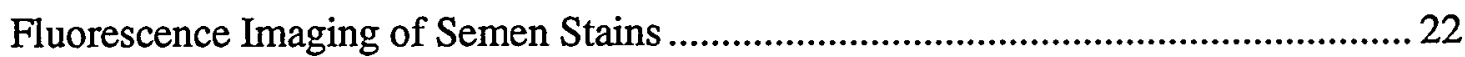

Test of Ambient-Light Rejection ......................................................................... 23

Comparison Test of Fluorescence-Imaging Techniques ........................................ 24

Exploration of Contrast-Improvement Methods with CLU...................................... 26

Time-Series Test............................................................................................. 27

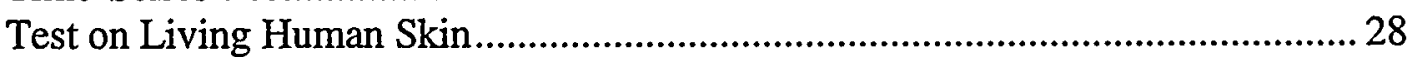

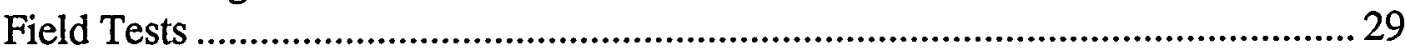

Reflectance Imaging of Bloodstains ......................................................................... 29

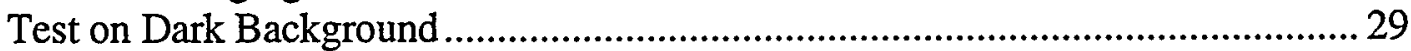

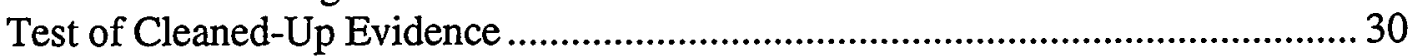

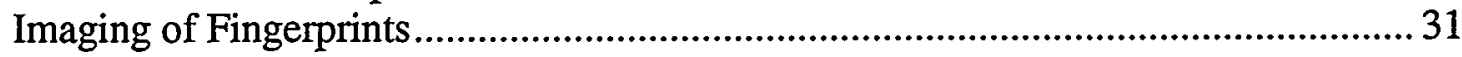

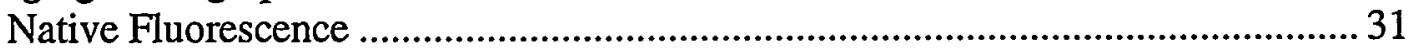

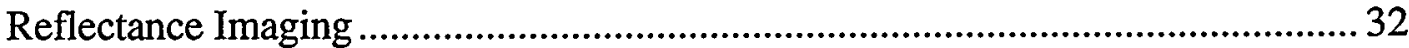

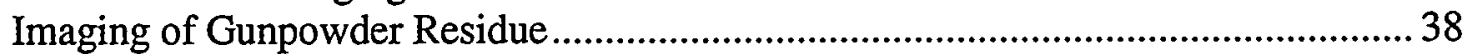

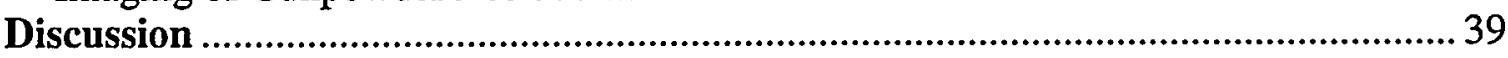

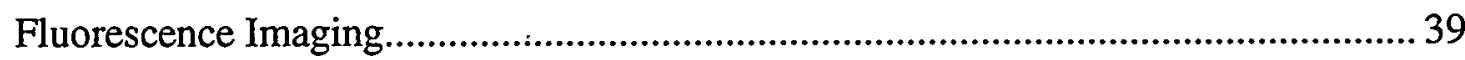

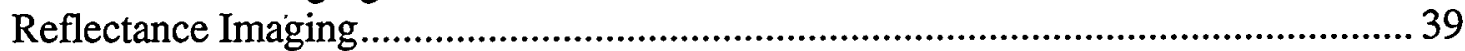

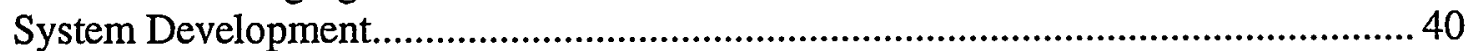

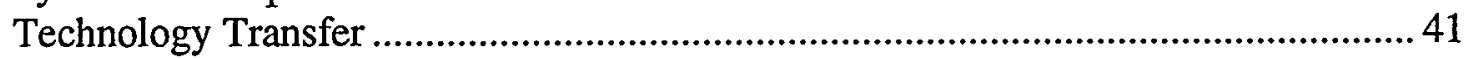

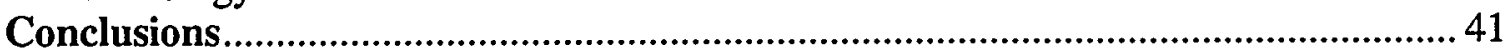

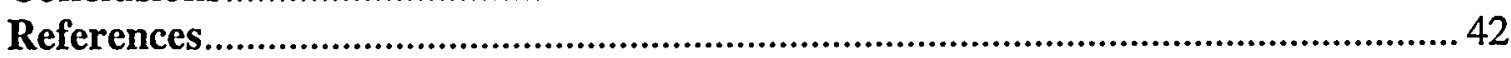




\section{Figures}

1 Typical Intensity Spectra (not to scale) of Excitation, Emission and

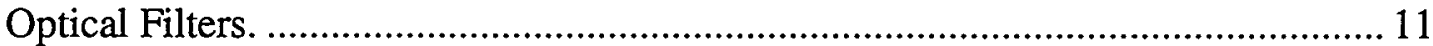

2 Photograph of CLU, Sandia's Multispectral Imaging System. ................................ 19

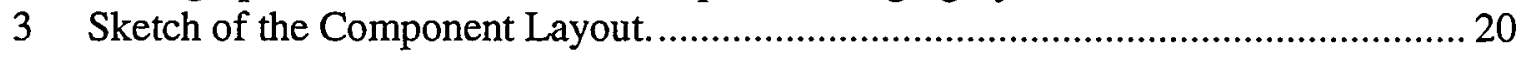

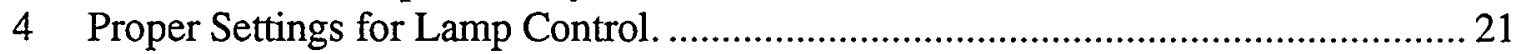

5 Correct Settings of Video and Camera Controls. ................................................... 21

6 CLU Images of a Semen Stain on 100\% Cotton Fabric (APD Control Sample \#1) with a) Room Lights On and b) Room Lights Off................................. 23

7 Semen-Stain Images of the APD Control Samples Taken by the Conventional Technique (Left) and Then by CLU (Right)...........................................................2 25

8 CLU Images of a Semen Stain on 100\% Cotton Fabric (APD Control Sample \#1) Taken with Different Filter Combinations. ............................................ 26

9 CLU Images of a Semen Stain on Upholstery Fabric (APD Control Sample \#3) with Various Emission Passbands........................................................ 27

10 CLU Images of Fluorescence of the Same Semen Stain (APD Control Sample \#1) over a Two-Month Period................................................................. 28

11 CLU Image of Semen Fluorescence on Living Human Skin................................28

12 Photograph (Left) and CLU Image (Right) of Dried Bloodstains on a Piece

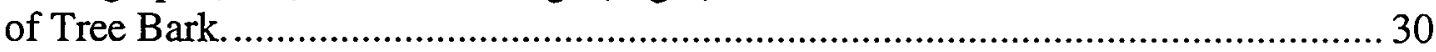

13 Photograph (Left) and CLU Image (Right) of Cleaned Bloodstains on a Rock........ 30

14 Fluorescence Spectra from Untreated Fingerprints. ............................................... 31

15 Reflected White-Light Image of a Fingerprint on a Glass Slide................................ 32

16 Photo of Plastic Ruler and Images of Two Fingerprints Captured from Ruler. ........ 34

17 Fingerprint Images Captured from Near and Far Side of Pictured Pistol. ................. 34

18 Fingerprint Image Captured from Plastic Security Badge. .........................................35

19 Photo of White Plastic Sample and the Fingerprint Image Captured from the

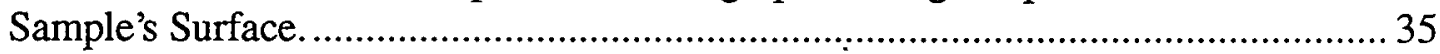

20 Photo of Formica Sample and the Fingerprint Image Captured from the

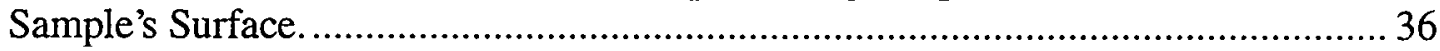

21 Photo of Rough Formica Sample and the Fingerprint Image Captured from

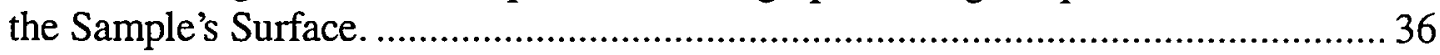

22 Photo of Computer Floppy and the Fingerprint Images Captured from the

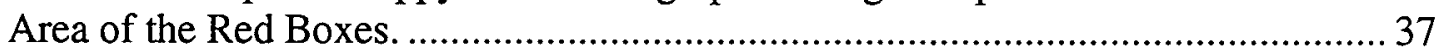

23 Reflectance Image of a Fingerprint on a Curved Knife Blade................................ 37

24 Transmission and Reflectance Spectra of Cotton Containing GSR Relative

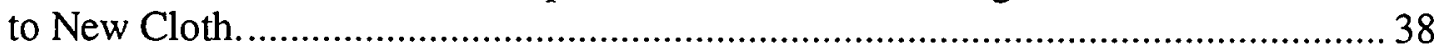

\section{Tables}

1 System Components and Manufacturers. ............................................................ 18

2 Characteristics of Semen-Stain Control Samples Provided by APD Crime Lab ...... 23

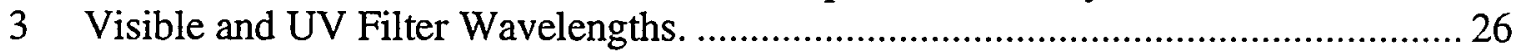

4 Filters Used to Limit the Wavelengths Seen by the Camera. ................................... 27 


\section{Acronyms and Abbreviations}

$\begin{array}{ll}\text { APD } & \text { Albuquerque Police Department } \\ \text { AFIS } & \text { Automated Fingerprint Identification System. } \\ \text { CCD } & \text { charge coupled device } \\ \text { CLU } & \text { Criminalistics Light-imaging Unit } \\ \text { CRADA } & \text { Cooperative Research and Development Agreement } \\ \text { DPSS } & \text { diode-pumped solid state } \\ \text { GSR } & \text { gunshot residue } \\ \text { I/O } & \text { input/output } \\ \text { IR } & \text { infrared } \\ \text { J } & \text { joule } \\ \text { LCD } & \text { liquid crystal display } \\ \text { mJ } & \text { millijoule } \\ \text { mm } & \text { millimeter } \\ \text { MTI } & \text { Molecular Technologies, Incorporated } \\ \text { NIJ } & \text { National Institute of Justice } \\ \text { nm } & \text { nanometer } \\ \text { OD } & \text { optical depth } \\ \text { RUVIS } & \text { Reflective Ultraviolet Imaging System } \\ \text { Sandia } & \text { Sandia National Laboratories } \\ \text { SBW } & \text { spectral bandwidth } \\ \text { SWP } & \text { short-wave pass } \\ \text { UV } & \text { ultraviolet } \\ \text { VCR } & \text { video cassette recorder } \\ \text { 3-D } & \text { three-dimensional } \\ \mu \text { S } & \text { microsecond } \\ & \end{array}$


This page intentionally left blank. 


\section{Executive Summary}

Crime ranks as one of the leading concerns of the American public. Despite downward trends in the rates of violent crimes and property crimes during the late 1990s [1], crime touches the lives of millions of people. In 1997, for example, findings from the National Crime Victimization Survey indicated that U.S. residents age 12 and older experienced approximately 34.8 million crimes [2]. The magnitude of the crime problem places great pressure on law enforcement, always operating within constrained resources, to apprehend offenders and bring them to justice. One possible solution to alleviating crime and the concerns of the American public is the development and deployment of new technology that speeds up the investigative process and improves the accuracy of the obtained evidence. Consequently, officers could investigate more crime scenes and the evidence gathered would provide greater certainty that those who commit crimes would be apprehended and brought to justice.

Current forensic methods used by law enforcement for gathering evidence at crime scenes are encumbered by a number of difficulties. Though these techniques do allow for the detection of evidence, the time required to process the large area of a crime scene is significant. This problem is further compounded by the limited number of available hours and the large number of crime scenes. Investigators must therefore limit the areas of their searches in individual investigations, as well as the number of investigations that can be conducted. Furthermore, certain methods, such as those that employ fluorescence, are often ineffective in capturing evidence during daylight hours. And other methods, like some of those used to detect latent fingerprints, require the use of chemicals and/or special equipment that can pose safety risks to those who employ the techniques and potentially to any bystanders, such as crime victims. There are also concerns that the implementation of certain forensic methods contributes to contamination of the crime scene and/or to damage of the evidence. And because the true amount of potential evidence at a crime scene is unknown, it is impossible to evaluate the sensitivity of current evidence-detection methods.

To address the concerns of the public in general and of law enforcement in particular, Sandia National Laboratories (Sandia) has developed a prototype multispectral imaging system for the National Institute of Justice (NIJ). This system, called the Criminalistics Light-imaging Unit (CLU), enhances investigators' ability to find fluorescing evidence at the scene of a crime and demonstrates the potential to visualize other types of evidence. CLU employs state-of-the-art technology that provides for viewing and recording of the entire search process on videotape, with output in a form that can be presented as evidence in a courtroom. Unlike some of the other current technologies and methods, CLU is safe to use and does not cause damage to potential evidence. 


\section{Project History and Goals}

CLU was developed under a contract with NIJ that was initiated in October 1997 and completed in April 1999. During all phases of the project, titled "Fluorescence Imaging Tools for Law Enforcement," Sandia worked closely with the Albuquerque Police Department (APD) Crime Lab and our commercial partner, Molecular Technologies, Inc. (MTI). This company was selected in a nationwide search based on its ability to bring the new technology to market.

Sandia's initial goal for this project was to develop fluorescence imaging tools that were capable of locating body fluids evidence at crime scenes under normal lighting conditions. This goal translates into the technical objectives of rejecting ambient light and improving sensitivity and specificity. The approach proposed to meet these objectives was a strobed light source and synchronized shuttered viewing glasses. Sandia built a prototype of this simple system but the system's performance did not meet the technical objectives. Subsequently, with the support of the APD Crime Lab, Sandia identified additional needs of local law enforcement and enhanced the initial project goal to include the visualization of multiple types of evidence, which also required revising our initial approach. As part of this evaluation process, Sandia spent over 40 hours in the field with APD personnel as observers at various crime scenes and an additional 20 hours observing laboratory techniques. A number of design considerations were evaluated to develop an imaging system that would be capable of visualizing multiple types of evidence. The new multispectral imaging system, CLU, was built and tested at Sandia and underwent a number of evaluation tests with APD. This system was also tested in the field as part of an active APD murder investigation.

\section{System Capabilities}

CLU meets the initial project goal of enabling investigators to find fluorescing evidence at the scene of a crime in normal lighting conditions. Operationally, CLU, which is camera-based, works at multiple wavelengths and demonstrates the ability to reject ambient light by a factor of approximately 100 . Improvements in sensitivity over current methods are estimated to be a factor of 5. CLU also supports the enhanced project goal of identifying other types of evidence such as blood patterns and, to a limited extent, untreated fingerprints. These other types of evidence are identified through a process called reflectance imaging - a detection process that emerged as an unpredicted capability during the system development process. In addition, CLU's analog videorecording feature allows investigators to visualize and highlight individual images of suspected evidence at any time during the search process.

Evaluation tests with APD pointed out the effectiveness of CLU in detecting and documenting evidence on different substrates. The most convincing result occurred, however, during field testing of the system in an active murder investigation. CLU was able to locate semen stains on the skin surface of a woman's body that had been decomposing for four days. APD investigators were unable to detect any semen evidence 
using a conventional fluorescence technique. APD investigators recently informed Sandia that CLU's findings might help them solve the murder.

\section{Future System Development}

In its present form, CLU is not optimized for fieldwork. Rather, it is a prototype design that was developed to test and demonstrate a comprehensive set of capabilities. At the end of the development process, we realized that the capabilities would be more useful to law enforcement if they were available as two separate instruments. The first instrument would be a full-blown version of the current system, though in a more compact form. It would perform both fluorescence and reflectance imaging. The second instrument would be a handheld version that would perform only reflectance imaging. Efforts to further develop and commercialize these proposed instruments are ongoing.

\section{Document Overview}

This report describes the work performed by Sandia to design, build, test, evaluate, and commercialize CLU. The Background section provides a general overview of selected technologies and methods for evidence detection and documentation in crime scene investigations that motivated aspects of the system's design. Next, in the Method section, we describe the technical approach for achieving the project goals, the design considerations evaluated for implementing the approach, the system configuration, and the procedures for system operation. The Results section, which follows, provides a detailed set of images captured by CLU in APD evaluation tests to illustrate its advantages over current technologies and methods. In the subsequent Discussion section, we evaluate the capabilities of CLU to meet the project goals, identify possible future system development, and describe our efforts to move the system into the commercial marketplace and identify other potential system applications. Finally, in the Conclusions section, we summarize the technical approach, results, and potential impact on law enforcement.

\section{Background}

Part of the system design process involved learning about technologies and methods that are available to local law enforcement for detecting and documenting forensic evidence in the field and in the laboratory. During this process, the needs for improved approaches in gathering three types of evidence were identified: semen stains, blood spatter patterns and trails, and fingerprints. In this section, we discuss some of the conventional and experimental technologies and methods for detecting and documenting these evidence types and highlight difficulties with their use. Our understanding derives in large part from interviews with APD Crime Lab personnel, supplemented by our experience and additional research. The information we gained throughout the system design process was useful in determining the set of capabilities that the imaging system should exhibit to meet the needs identified by local law enforcement. 


\section{Semen Stains}

The primary evidence sought by police investigators at the scene of a sexual assault is a semen stain. With the advent of DNA fingerprinting, a semen sample becomes a valuable piece of evidence. Before the DNA can be analyzed, however, the semen stain must be located and sampled. The conventional method used by local law enforcement for locating semen stains employs fluorescence because of the naturally fluorescent properties of semen in the visible region of the optical spectrum.

Fluorescence is a process that begins with the electrons of a molecule being excited to a higher energy state by the absorption of light. The absorption of light is often termed excitation in the context of fluorescence. Because the electrons prefer to minimize their energy levels, the absorbed light is released in the form of heat or an emitted photon. A photon is a term for a unit of optical energy. The emitted photon is referred to as fluorescent light. The wavelength of the fluorescent light is longer than the excitation photon since the excitation and emission process uses some energy. Wavelength refers to the color of light absorbed and emitted. For instance, semen absorbs blue light and gives off green to yellow light. Most organic molecules exhibit some fluorescence. The percentage of absorbed photons converted to fluorescence is dependent upon the electronic bond structure of individual atoms in the molecules. In the case of semen, the percentage is significant and fluorescence can be used to locate otherwise invisible stains.

The conventional fluorescence method for locating semen stains at a crime scene is to illuminate the area with the light from a high-intensity lamp, termed an alternative light source, while viewing the illuminated area through optical filter glasses. Typically, an arc lamp is used with a flexible light guide to illuminate the area. An optical filter is placed in front of the lamp to limit the spectral bandwidth (SBW) of the excitation light to the peak absorption wavelength for semen. The wavelength relationship between the optical filters and fluorescence spectra is illustrated in Figure 1. The primary reason for limiting the SBW is to excite fluorescence from the evidence while blocking the lamp wavelengths that overlap with the emission wavelengths. A secondary reason for limiting the SBW is to minimize the amount of background fluorescence from the other fluorophores, which are substances that fluoresce. The filter glasses worn by the observer block the blue excitation light from the lamp while the emission wavelengths are being transmitted. Hence, the observer sees the dull glow of the fluorescence but not the bright excitation light from the lamp. 


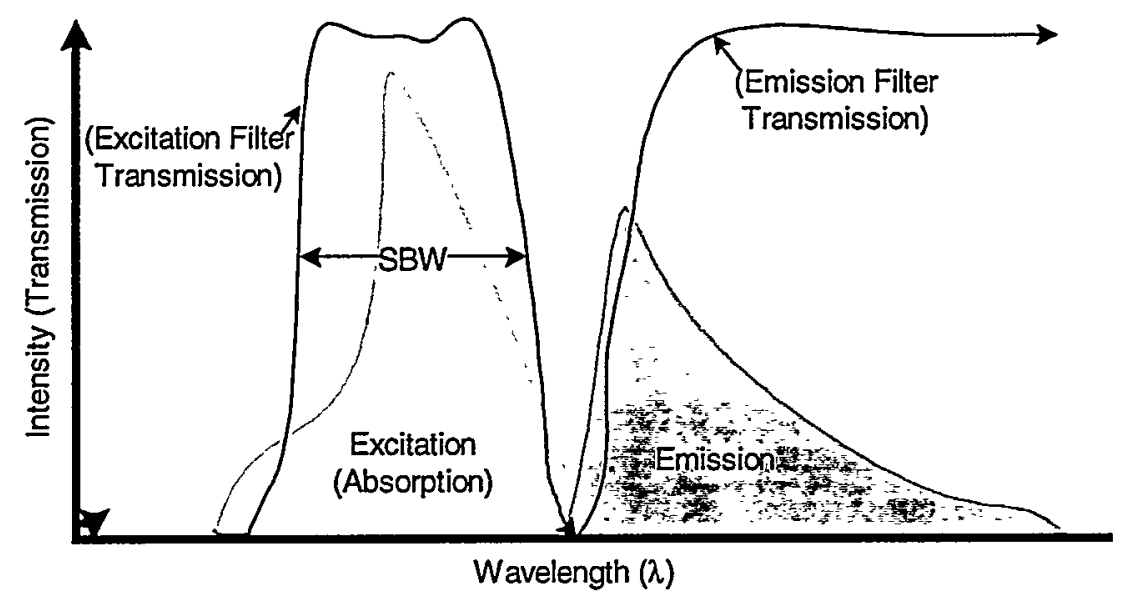

Figure 1. Typical Intensity Spectra (not to scale) of Excitation, Emission and Optical Filters.

The conventional fluorescence method for semen detection has a number of drawbacks. Though semen fluoresces, the light emitted by the semen is weak compared to ambient (i.e., surrounding) room light, which greatly hinders detection. If the crime scene is outdoors, the investigators must wait until nightfall to use the technique. If the crime scene is indoors, investigators must turn off all internal lights and black out the windows. Blacking out the windows entails not only additional time and effort, but it also introduces the possibility that those who perform this task will contaminate the crime scene. And even when a room has been blacked out, investigators often complain that many other substances besides semen fluoresce, like food spills and animal urine. Because of the multiple-substance-fluorescing problem, investigators often collect all the questionable fluorescing substrates, such as entire bed sheets and couch cushions, to complete the search in a reasonable time. The task of detecting and documenting semen stains is then transferred to technicians at the crime lab.

In those cases where investigators do detect evidence that they suspect contains semen stains, it would be ideal to photograph the evidence. However, setting up a 35millimeter $(\mathrm{mm})$ camera at a crime scene to take a number of time exposures is so time consuming that investigators often do not bother to photograph the evidence. And even if such evidence is photographed at the scene, there is no assurance until the film is developed that the photographs have evidentiary value.

APD investigators rarely use alternative light sources in the field, principally because of background fluorescence, the need to black out windows, and the weight of the fluorescence-imaging equipment (about 35 pounds). It is common practice for APD investigators to take everything that might contain semen evidence to the crime lab for subsequent examination. Selecting all potential evidence results in a large number of samples from each scene and uncertain sampling efficiency. This practice also results in additional detection and documentation work for technicians in the crime lab. 


\section{Blood Spatter Patterns and Trails}

Investigators often reconstruct a crime from blood spatter patterns and blood trails, both of which are very difficult to visualize on dark surfaces. For example, in the case of a shooting that is committed indoors, investigators have no easy way to document blood spatter patterns on dark paneling or carpet. That information would be particularly important when, for instance, one individual involved in the incident claims that he shot the other in self-defense, but the blood spatter patterns, if detectable, were to indicate that the victim was 15 feet away when shot and thus not a threat. Similarly, in the case of an individual who is shot and runs several blocks before collapsing and dying, investigators have difficulty in locating the blood trail that leads to the shooting and no way to document the trail as evidence if it is on dark pavement. The investigator's work is further complicated when blood evidence has been cleaned up to conceal a crime.

APD investigators use the chemical reagent luminol to reveal blood spatter patterns and/or to obtain samples of the blood. Luminol is applied to the area of interest via a spray bottle. When it encounters blood, luminol reacts and phosphoresces, i.e., produces a faint glow.

Several limitations are associated with the use of luminol. As with the fluorescence method for semen stains, blood treated with luminol produces such a faint glow that it is not only difficult to see but also difficult to photograph. To create the requisite timeexposed photographs, investigators must either wait for (or create) a dark environment. The luminol method is also cumbersome and expensive to use for blood patterns or trails that cover large areas or that are on dark surfaces. In the latter case, a lot of the chemical reagent may be used (and wasted) simply in locating all of the blood evidence. Schiro [3] contends that spraying luminol at crime scenes should be an investigator's last resort for detecting blood because it has several drawbacks as a presumptive test for blood. These problems include giving false reactions, causing the loss of several genetic markers, causing latent and possibly bloody impressions to smear, and rendering some dilute stains unavailable for further analysis.

\section{Fingerprints}

The primary evidence sought by police investigators at a crime scene is the offender's fingerprint. A variety of fingerprint detection methods are available to law enforcement currently, and forensic researchers are experimenting with newer methods to overcome some of the difficulties with current approaches. The conventional approaches to fingerprint detection employ physical and/or chemical development processes, which involve pretreatment of the evidence. In some cases, these processes are ineffective in visualizing fingerprints and require additional illuminating equipment such as a laser or alternative light source. Newer approaches such as those that employ inherent fluorescence and ultraviolet (UV) reflectance are being proposed as ways to capture fingerprints without pretreatment. In the subsections that follow, we examine characteristics and limitations of selected techniques employed in four methods of 
fingerprint detection: physical development, chemical development, fluorescence, and UV reflectance.

\section{Physical Development}

The time-tested physical-development method of finding latent fingerprints consists of dusting a crime scene with special powder and removing the prints with adhesive tape [4]. When conducting a search, investigators must weigh the time to dust and collect the possible prints against the time allotted to an individual crime scene. Oftentimes, the search is limited because the investigator finds nothing to dust and/or must quickly conclude the search to move on to waiting calls. To be time efficient, the investigator performs the following activities:

- Surveys the crime scene to determine which surfaces have the highest probability of containing fingerprints.

- Screens those surfaces (i.e., illuminates with a flashlight at an oblique angle) to find disturbed dust or changes in the surface reflectance shaped like a fingerprint.

- Dusts the identified positive areas with dusting powder.

- Evaluates the fingerprint ridge content, i.e., looks for approximately 10 points (identifying markers) on the fingerprint.

- Tapes, lifts, and adheres the viable fingerprint to a card and labels each card.

Fingerprint collection by dusting has a number of limitations. First, the process is both messy and cumbersome. All of the conventional powders leave surfaces difficult for crime victims to clean; investigators especially dislike silk powder because it is extremely easy to inhale. Second, as noted in the product literature [5], dusting is generally useful only on newer prints. And third, as demonstrated in experimental work by James et al. [6], fingerprints can be partially or totally obliterated by overpowdering and by overbrushing.

\section{Chemical Development}

Chemical reagents are often used to develop latent fingerprints. Situations that prompt the use of chemicals include those where the prints cannot be lifted by dusting, and those where the prints are of unknown age and are the object of investigation in a high-profile case-like a gun in a murder. While a variety of chemical reagents are available, we have chosen to focus on two techniques observed in our work with APD to identify potential problems that are inherent in the use of chemical treatments in general.

\section{Ninhydrin}

Ninhydrin has been described as one of the most affordable and useful reagents for visualizing latent prints on porous surfaces such as paper, wood, and walls [7]. This 
reagent reacts with the amino acid components of biological fluids to produce a purplecolored product called Ruhemann's purple [5]. Ninhydrin can be mixed with a carrier to form a liquid solution or it can be purchased premixed. The treatment is applied by spraying, brushing or dipping [8]. Development times of the treated prints vary based on such factors as the surface, the amount of amino acid, and the environment [9]. In some instances the development time can be as much as 10 days [5]. However, the development process can be accelerated, once the print is dry, in a humidified environment or alternately with a steam iron [8]. Ninhydrin may also be used as one of a sequence of methods for developing a specific print $[5,7,8]$.

A number of limitations of ninhydrin have been noted both in our review of the literature and in our observations at the APD Crime Lab. Four such limitations are recounted here. First, the carrier used to dissolve ninhydrin can be dangerous. For example, acetone-ninhydrin solutions are extremely flammable and hazardous to inhale [9]. Second, ninhydrin is not useful on certain items, like those that have been exposed to water [5] and paper that has high animal glue content [8]. Third, acetone-ninhydrin treatments can negatively affect subsequent examinations of the fingerprints. [9]. And fourth, as we observed in our work with APD, ninhydrin-treated fingerprints may not reveal a clear contrast between the print and its background surface. In such an instance, photographs must be taken of the fingerprints, which requires additional effort to work the image so that it is of sufficient quality for print digitization and subsequent submission to the Automated Fingerprint Identification System (AFIS) database.

\section{Cyanoacrylate Fuming}

A commonly used technique in the development of latent fingerprints is cyanoacrylate (Super Glue (B) fuming [10]. Cyanoacrylate fuming is useful on nonporous surfaces, as well as some slightly porous surfaces $[5,11]$. The vapors are adsorbed and polymerized on the surface under examination [12], causing latent print residues on such surfaces to appear white in color [11]. Cyanoacrylate fuming can be accomplished in a variety of ways (e.g., in a fuming chamber, with a fuming wand, or via fuming packets). During this project, we observed APD investigators using a wand under a fume hood to perform the technique on a pistol and on plastic baggies that were recovered in a drug raid. We also observed investigators dispersing cyanoacrylate from a small packet into a car that was involved in a murder.

Safety is a primary concern when performing the cyanoacrylate fuming technique. The fumes are strongly irritating to the eyes and respiratory system. Adequate ventilation is required and contact with the skin should be avoided [11]. In our work with APD, investigators who fumed several articles of evidence under a fume hood wore both safety glasses and gloves. There is also the possibility that certain methods of fuming run the risk of releasing deadly cyanide gas at high temperatures. German [13], for example, urges extreme caution when working with commercially available butane-fueled torch fuming kits. Another concern about cyanoacrylate fuming pertains to the results of treatment. Because cyanaocrylate-developed fingerprints are white, those that occur on light-colored backgrounds are difficult to see or photograph and their contrast may need to be enhanced by other methods [10]. APD investigators also find that some prints do 
not turn white, and their contrast must be enhanced with chemical treatments on any colored background.

\section{Fluorescence}

A common method used to enhance latent fingerprints is fluorescence detection [14]. One such detection technique that we investigated during the system design process was the use of fluorescent dyes to stain prints developed via cyanoacrylate fuming. According to Lock, Mazzella, and Margot [15], most fingerprint laboratories routinely use fluorescent dyes, such as Ardrox, Rhodamine 6G or Basic Yellow, to enhance or increase the sensitivity of detection of cyanoacrylate-pretreated fingerprints. However, as we learned, staining with a fluorescent dye and observing with room light is often insufficient to visualize the fingerprints. In such cases, an alternative light source or a laser is needed to illuminate the dyes and make the print visible. The process of detection then becomes evermore labor-intensive and potentially expensive. Not only must there be equipment available to illuminate the article of examination, but the illuminated evidence must be photographed - requiring the same time-exposure issues described previously for other types of treated evidence.

The ideal in fingerprint detection is a technique that captures a fingerprint without pretreatment, i.e., physical and/or chemical development. Such a nondestructive technique would save time, eliminate mess, save prints that are currently damaged by conventional treatments (either used separately or as a result of successive treatments), and avoid the safety hazards associated with many of the current techniques. In addition, a technique that requires no pretreatment could potentially capture a new class of prints that current techniques either do not develop or damage.

\section{Investigations into Using Inherent Fluorescence}

To achieve the ideal in fingerprint detection, forensic researchers have attempted to use the inherent, or native, fluorescence of fingerprints to make them visible. Experiments to capture the inherent fluorescence have been performed both in the visible region of the spectrum $[16,17,18]$ and in the ultraviolet (UV) region of the spectrum [14, $18,19]$. This work, though it has obtained some successes, has also demonstrated numerous difficulties.

Because the inherent fluorescence of fingerprints is very weak [16], special equipment is required to visualize the prints. This equipment may include a laser or strong, well-filtered lamp as the light source [16], and/or a scientific-grade chargecoupled-device (CCD) camera for the recording device as used in experimental work by Yosef et al [19]. From a practical perspective, the cost of equipment necessary to capture inherent fluorescence is very expensive for local law enforcement. In our experience, the lasers can cost between $\$ 20,000$ and $\$ 50,000$, and the scientific-grade CCDs can cost between $\$ 20,000$ and $\$ 40,000$. Such equipment may also be too complex and large for fieldwork. From a safety perspective, special procedures are required when using lasers and UV radiation. Protection of the eyes is necessary for laser work, and protection of the 
eyes and possibly skin is required to avoid exposure to the harmful effects of UV radiation.

Another difficulty in using inherent fluorescence is that even with special equipment, the technique may not work. As pointed out by Menzel [20], laser detection of fingerprints in the visible region of the spectrum is often not successful because of frequently occurring background fluorescence, which overwhelms the inherent fluorescence. In those cases, the inherent fluorescent technique must be supplemented by other pretreatment procedures to improve visibility under laser illumination [21].

A third difficulty in using inherent fluorescence is that detection is inconsistent. This inconsistency is found in studies performed in both the visible region and the UV region of the spectrum. Dalrymple et al. [16], for example, reported the detection of inherent fluorescence in the visible region of the spectrum using a laser coupled with photography. Mimicking the experimental configuration described by Dalrymple et al. [16], however, Salares, Eves, and Carey [17] found that only one-fifth of their population gave weakly luminescent fingerprints that were only detectable under close scrutiny; the remaining fingerprints $(80 \%)$ were undetectable. In comparing the use of inherent fluorescence in the visible and UV regions of the spectrum, Bramble et al. [18] found that UV fluorescence can be used to detect many fingerprints that escape detection by visible fluorescence. These researchers were able to detect $69 \%$ of latent fingerprints using 266 $\mathrm{nm}$ radiation from a Nd:YAG laser and UV optics, compared to a detection rate of $23 \%$ using an argon-ion laser at $514 \mathrm{~nm}$. Yosef et al. [19] also investigated using UV fluorescence for latent fingerprint detection, with a setup composed of a mercury-xenon lamp and a CCD camera - both of which were water-cooled and computer-controlled. The detection rate for their fingerprint sample, however, was only $10 \%$.

A fourth difficulty relates to the consequences of subjecting fingerprints to UV illumination. As Bramble et al. [18] emphasize, further research is needed to assess the effects of UV fluorescence on subsequent analyses of the evidence, especially DNA profiling, which is known to be susceptible to damage by UV photography.

\section{UV Reflectance}

Researchers have also been exploring an imaging technique known as UV reflectance. According to Krauss and Warlen [22], fluorescent photographic techniques record visible light generated by the UV radiation of an object, whereas reflective photographic techniques record an object's reflection and absorption of UV light. Experimental work performed by Keith and Runion [23] using the U.S. Army's Reflective UV Imaging System (RUVIS) indicates that this technique is valuable in locating and photographing latent fingerprints on multicolored backgrounds that were undetected, or detected but considered unsuitable, by other techniques such as laser illumination and direct-reflection white-light examination. The Army's RUVIS was composed of the following components: 1) a pocket-scope type night-viewing device sensitive at short-wave UV wavelengths, 2) a solar-blind/short-wave UV transmitting filter, 3) a short-wave UV transmitting (quartz) camera lens, and 4) a short-wave UV light source. 
Safety, equipment, and treatment-related concerns point out some of the difficulties associated with this technique. Regarding safety, UV reflectance imaging, like the inherent UV fluorescence technique, uses UV light. As described previously, using UV light presents significant safety risks. Not only do users have to wear safety glasses, but they also may have to cover their skin. Regarding equipment, the cost and complexity of UV-capable optics and sensors is significant. One commercial system is approximately $\$ 20,000$ [5]. With respect to treatment of evidence, the success of UV reflectance imaging as a solution that bypasses pretreatment by physical and/or chemical means is not assured. For example, the RUVIS results reported by Keith and Runion [23] were based on pretreated cyanoacrylate-fumed prints, and product literature on some commercial systems either 1 ) indicates that such fuming is required when preliminary examination with the UV reflectance imaging system yields no results [24] or 2) claims that their system gives exceptional reproduction on cyanoacrylate-fumed prints [25]. Hence, for the reasons of safety, equipment cost and complexity, and need for possible pretreatment, the potential of UV reflectance imaging as the ideal in fingerprint detection has yet to be achieved. 


\section{Method}

The initial goal of Sandia's project with NIJ was to design fluorescence imaging tools that reject ambient light and improve the sensitivity and specificity of fluorescence to the target substance. With improvements in sensitivity and specificity, smaller signals from the target substance can be detected and distinguished from background substances that also fluoresce. The initial goal was enhanced during the project to include the capability of visualizing other types of evidence such as blood spatter patterns and trails, and untreated fingerprints. This section discusses the technical approach followed to achieve the project goals, explains how we selected the components to implement the approach, and describes the system configuration of the Criminalistics Light-imaging Unit (CLU) and the proper procedure for operating CLU.

\section{Technical Approach and Design Considerations}

The descriptions of the system technical approach and the design considerations involved in the selection of major system components have been removed from this version of the report to protect the business interests of the corporate partner. This information may be released at a later time when the technology is patent protected and on the market.

\section{System Description}

Table 1 identifies all the system components that were used in CLU. Copies of component manuals are contained within the delivered system.

Table 1. System Components and Manufacturers

\begin{tabular}{|l|l|l|}
\hline Component & Model Number & Manufacturer \\
\hline Intensified CCD camera & ISG-250 & Xybion, San Diego CA \\
\hline Video control unit & VIS-100 & Xybion, San Diego CA \\
\hline Camera control & CCU-01 & Xybion, San Diego CA \\
\hline Pulsed arc lamp & 60000 & Oriel, Stratford CT \\
\hline Lamp control unit & 68826 & Oriel, Stratford CT \\
\hline Filters & Various & Omega Optical, Brattleboro VT \\
\hline Lenses & Various & Oriel, Stratford CT \\
\hline VCR/LCD screen & GV-A500 & Sony \\
\hline Light Guide & LLG-5MM & Oriel, Stratford CT \\
\hline
\end{tabular}

A picture of CLU is shown in Figure 2, using a 12-inch ruler for scale. 


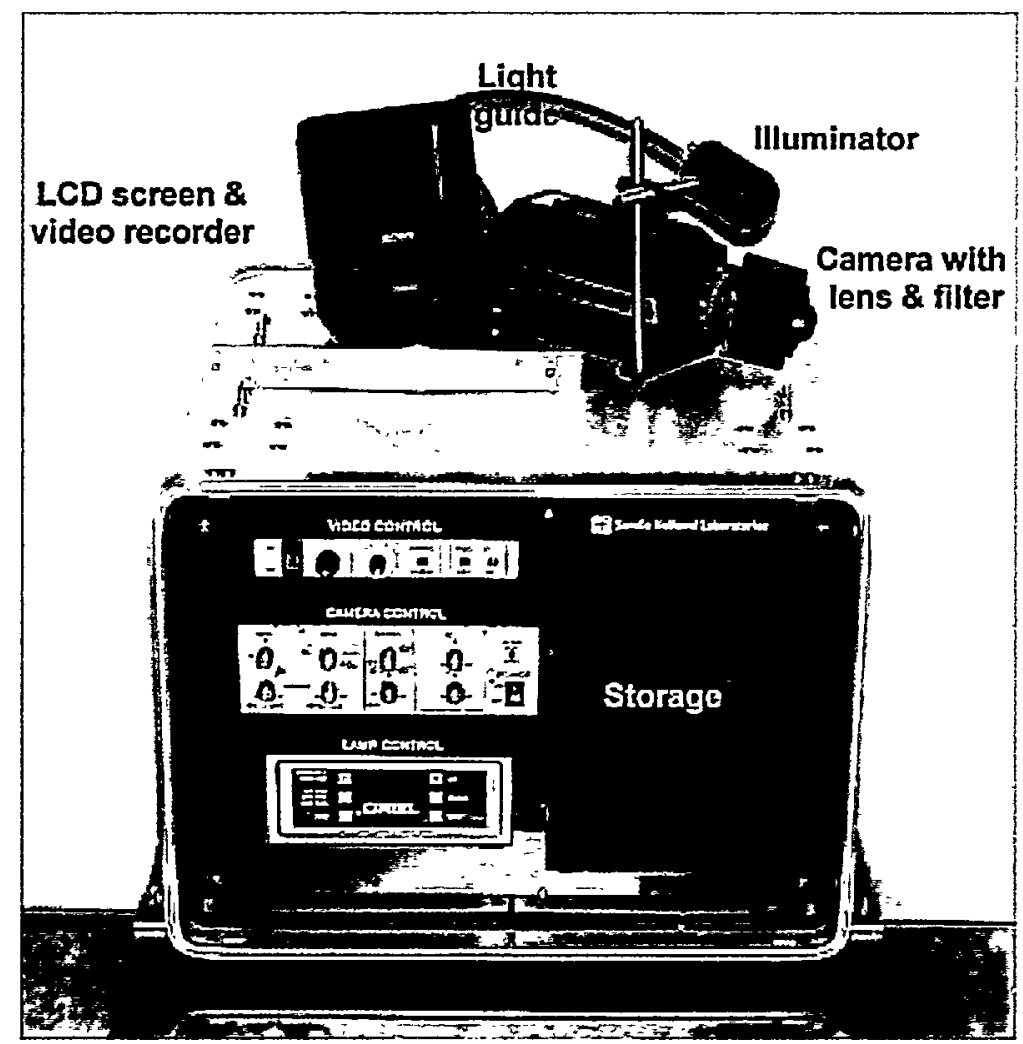

Figure 2. Photograph of CLU, Sandia's Multispectral Imaging System.

The arc lamp and control units for the lamp, camera, and video output are housed in a rugged travel case. When in use, the camera, video recorder, and illuminator are mounted on a tripod. A 10-foot cable carries the lamplight, power, and video signal between the case and tripod. The lamplight is transferred to the illuminator lens via a liquid light guide. This light guide is similar to a fiber optic, except that it is filled with a liquid rather than glass. The user should avoid bending the light guide into a bend radius less than 10 inches or stepping on it. The diameter of the lamp light on the scene is matched to the camera field-of-view by adjusting the distance between the light guide and the illuminator lens. The camera body contains an image-intensifier tube bonded via a fiber optic taper to a CCD image sensor. A conventional camera lens focuses the filtered light from the scene onto the image intensifier tube. A filter adapter and filter are threaded onto the front of the camera lens. One-inch-diameter interference filters are provided for each type of evidence. The light from the intensifier is captured by the CCD sensor and transmitted via standard video signal to an $8 \mathrm{~mm}$ video recorder. Two camera lenses are currently used with the system. Both lenses are attached via a standard $\mathrm{C}$-mount. When not in use, all of the tripod components are stored within the case and the cable is wound on the back panel. Covers are placed over the front and rear of the case for transport.

The layout of components in the case is shown in Figure 3. Light from the pulsed arc lamp is pseudo-collimated onto an interference filter by a condensing lens. Lamp filters are held in a cassette, which is labeled for wavelength. The filter holder is accessed through a small door in the back panel. A second lens is used to focus the filtered light 
from the lamp into the liquid light guide. The focus of the condensing and light-guide lenses can be adjusted to maximize the optical power from the light guide.

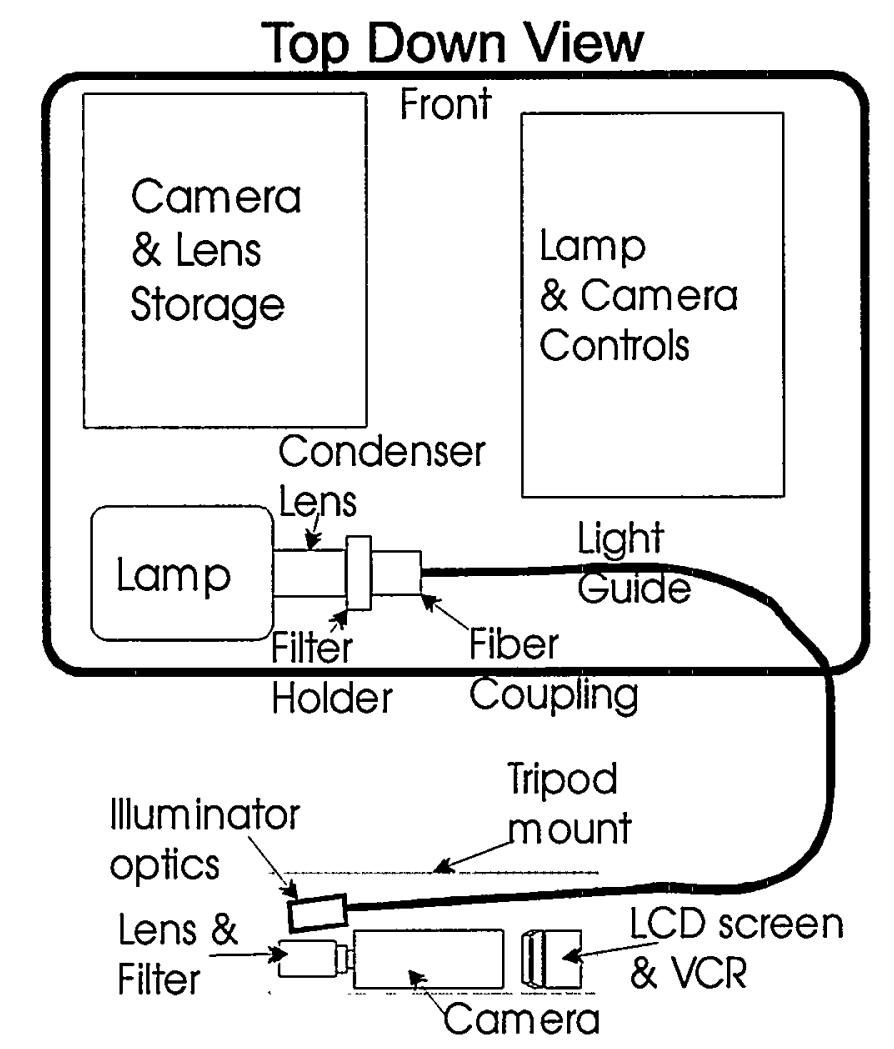

Figure 3. Sketch of the Component Layout.

All of the control units are plugged into a common power strip with an on/off switch mounted on the back panel. The power adapter for the VCR is mounted within the case, and a low-voltage supply line is run to the VCR on the tripod. The video signal from the camera is returned within the input/output $(\mathrm{I} / \mathrm{O})$ line to the video control unit. The output from the video control unit is returned to the VCR via a 75-ohm coaxial cable. The gate trigger of the intensifier is coupled to the lamp pulse by a conductor wrapped around one of the lamp supply wires.

\section{System Operation}

This subsection describes the proper procedure for operating the system. The procedure explains how to use the lamp, camera, and video controllers; it also briefly covers usage of filters and lenses.

The lamp controller, shown in Figure 4, allows the user to adjust the lamp power, repetition rate, and trigger source. The lamp trigger is first set to the external source ("EXT TRIG"). The repetition frequency is then determined by the external source and not by the value on the lamp controller. The lamp energy can be varied, but it is typically 
set via the UP button to the maximum of 800 millijoules $(\mathrm{mJ})$ for $60 \mathrm{~Hz}$ operation. The system is started and stopped by pressing the RUN button.

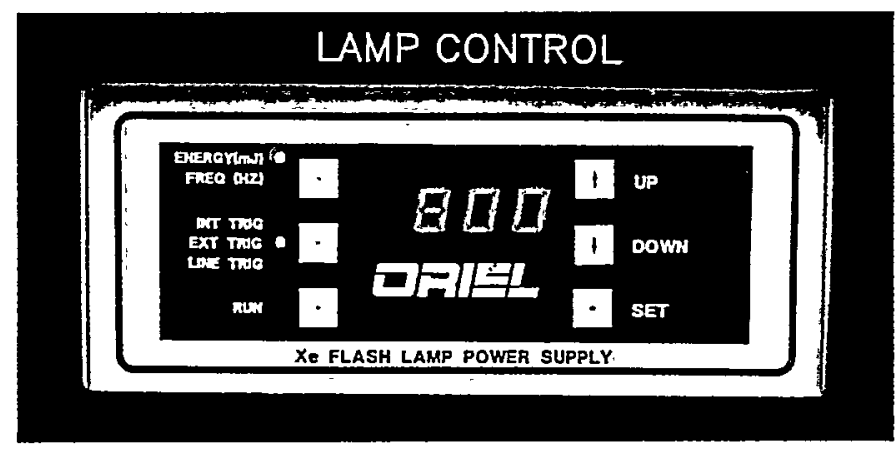

Figure 4. Proper Settings for Lamp Control.

On the camera controller shown in Figure 5, the gate trigger of the intensifier (TRIGGER SOURCE) is set to an external source ("EXT") and the gate duration is set to the RANGE of "1-10 $\mu \mathrm{s}$." The term gate refers to the time the intensifier is on and collecting light. When set properly, the MANUAL GATE and SLOW GATE lights will be on. The black arrow on the SLOW GATE Duration indicates the exact position of a 10 $\mu$ sate; however, the gate can be increased to full range to capture the tail end of the lamp pulse. When the lamp is not running, the MISSING EXT(ernal) TRIGGER light will flash and the gate will trigger with each video frame to allow the user to observe the scene through the camera. The brightness of the scene can be controlled by the INTENSIFIER GAIN in either the "AUTO" mode or manually. Reducing the aperture of the camera lens can reduce the brightness.

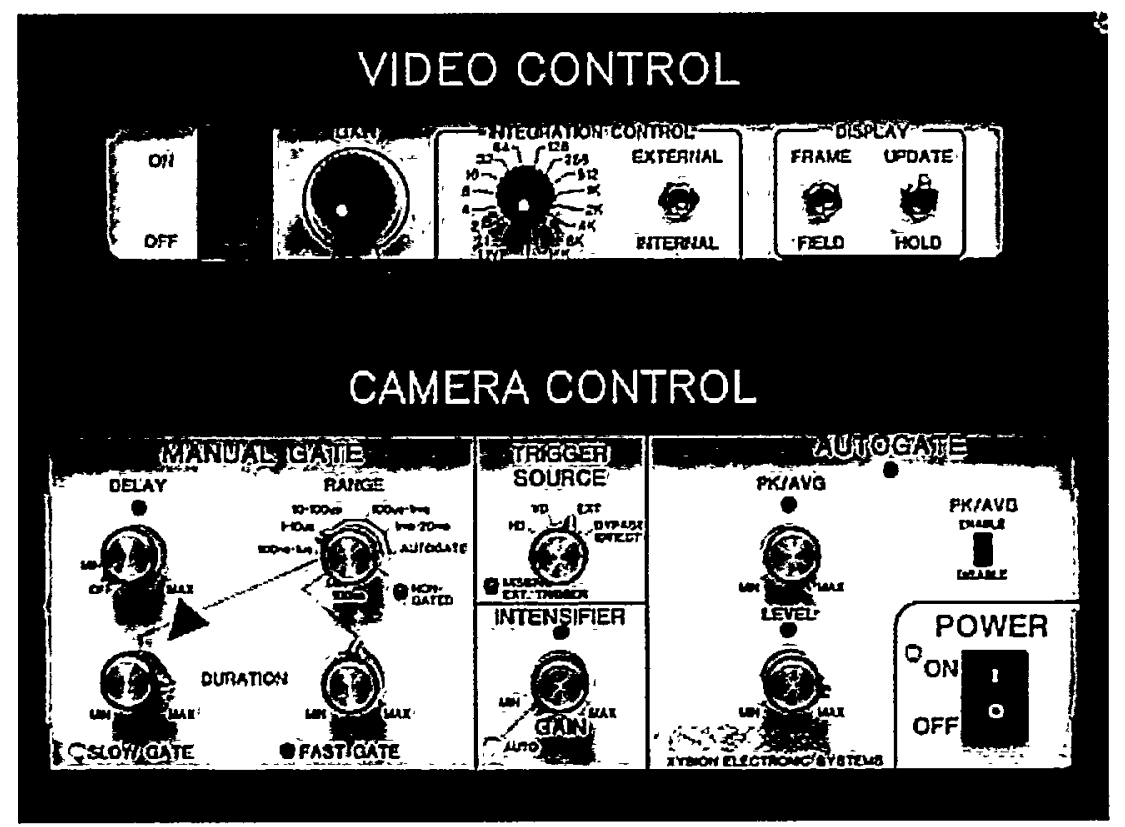

Figure 5. Correct Settings of Video and Camera Controls. 
The video controller, also depicted in Figure 5, lets the user integrate multiple images and freeze a video image. Because fluorescence is typically a weak optical interaction, it may be necessary to add, or integrate, the light collected from multiple images to form an image with acceptable contrast. The number of images integrated is selected in multiples of two, with the INTEGRATION CONTROL switch set to "INTERNAL." The maximum number of images integrated is limited by the dark noise of the camera, which becomes significant between 64 and 128 frames. As the intensifier gain is increased to its upper limit to capture weak florescence, so is the inherent intensifier noise. Thus, if the camera and sample can be held stationary, increasing the integration is the preferred method to increase brightness while avoiding the additional noise. Typically, the video GAIN is left at the minimum value, which is a factor of one. In the search application, it is convenient to freeze an image of interest rather than trying to hold the camera or sample still. The DISPLAY switch is toggled from "UPDATE" to "HOLD" to freeze the image on the screen. The other DISPLAY switch is set to "FIELD" since the camera is providing a field for every lamp firing.

Before collecting an image, the appropriate filter is threaded onto the camera lens and placed in the lamp filter holder. The filter for each application is described in the Results section, which follows. The lamp filter is accessed through the back panel door, and the filter cassette is ejected by pressing on the rod protruding from the rear of the holder. For viewing relatively large areas with visible light, the $50 \mathrm{~mm}$ focal length lens is used. For observing fine detail at high magnification (fingerprints), the $105 \mathrm{~mm}$ lens is used. The $105 \mathrm{~mm}$ lens is also UV capable and should be used for wavelengths below $400 \mathrm{~nm}$.

\section{Results}

This section describes the results obtained with CLU in imaging semen stains, blood stains, and fingerprints. Most of these results were demonstrated in evaluation tests conducted with the APD Crime Lab and are included here to illustrate the potential of Sandia's system to improve the evidence search process. Additional results from experimental work in imaging gunpowder residue are also discussed.

\section{Fluorescence Imaging of Semen Stains}

We used semen stains to evaluate CLU's fluorescence-imaging capability, given that locating semen stains appears to be the dominant application of fluorescence imaging in field investigations. The second popular application is imaging fingerprints treated with fluorescent dye. Although not imaged in this study, the results indicate the potential improvement for imaging treated fingerprints as well, since the dye fluorescence is strong compared to native semen fluorescence.

A number of semen-stain control samples were provided by the APD Crime Lab to evaluate CLU's abilities to reject ambient light and to improve sensitivity and specificity of fluorescence imaging to the target substance. The samples were prepared on several 
fabrics, some of which had been exposed to various chemical treatments such as bleach. The APD serologists chose fabrics and treatments known to be difficult to image with the conventional technique. Table 2 identifies the samples and their corresponding fabrics and treatments. Following this table are descriptions of the tests and exploratory work performed to evaluate CLU's fluorescence imaging capabilities on semen stains. (Please note that the samples were not used in all of the evaluation tests.)

\section{Table 2. Characteristics of Semen-Stain Control Samples Provided by APD Crime Lab}

\begin{tabular}{|c|l|}
\hline $\begin{array}{c}\text { Control } \\
\text { Sample }\end{array}$ & Fabric and Treatment \\
\hline 1 & $100 \%$ cotton, washed, bleached repeatedly \\
\hline 2 & $50 \%$ cotton and 50\% polyester tee-shirt, washed repeatedly \\
\hline 3 & Upholstery of unknown fiber \\
\hline 4 & Nylon carpet \\
\hline 5 & $95 \%$ cotton and 5\% Lycra stretch, washed once \\
\hline 6 & $100 \%$ polyester with shiny surface, washed once \\
\hline
\end{tabular}

\section{Test of Ambient-Light Rejection}

Figure 6 demonstrates the ability of CLU to reject ambient light using control sample \#1. The "lights on" image was taken in a room well-lit by fluorescent lighting. Because turning the light on or off has no effect on the brightness, the ambient-light rejection is working as expected. We estimate that CLU reduces ambient light by a factor of 100 .

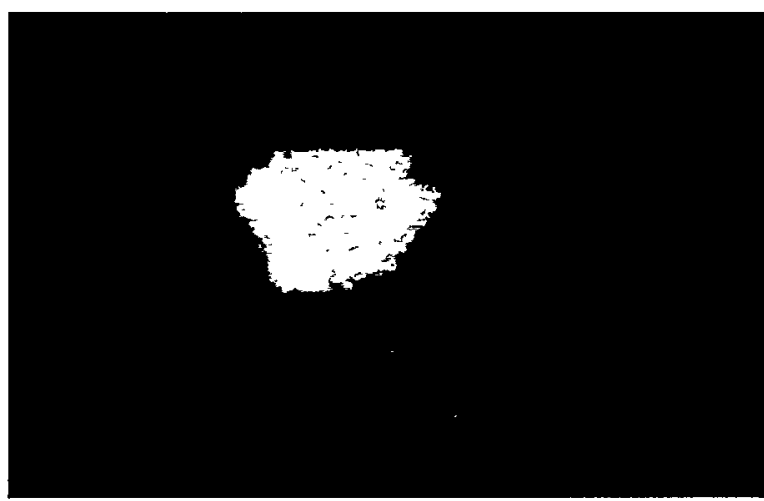

a)

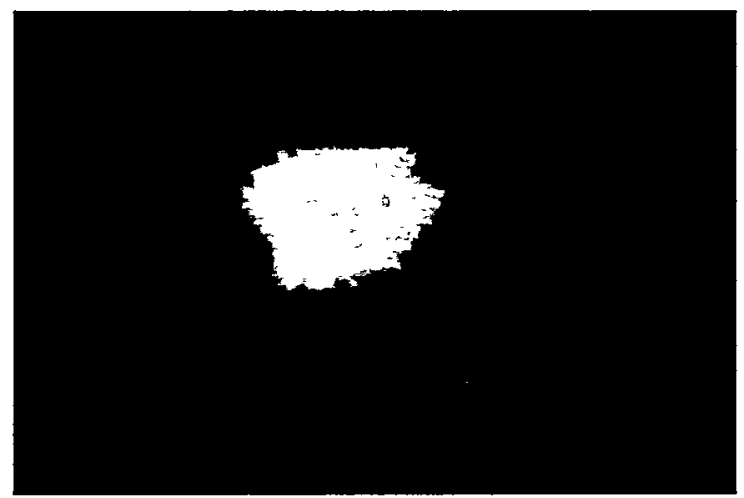

b)

Figure 6. CLU Images of a Semen Stain on $100 \%$ Cotton Fabric (APD Control Sample \#1) with a) Room Lights On and b) Room Lights Off. 


\section{Comparison Test of Fluorescence-Imaging Techniques}

Sandia and the APD Crime Lab conducted a test to compare images produced by the conventional fluorescent-search technique with those produced by CLU. In this test, we used all of the semen-stain control samples listed in Table 2. The APD Crime Lab first imaged the control samples with the conventional technique, and then the Sandia investigators imaged the control samples again with CLU.

The conventional technique consisted of a commercial arc lamp (sold as an alternative light source) that was filtered to emit blue light $(450 \mathrm{~nm})$. The lamp illuminated each sample and the fluorescence emission was captured by a $35 \mathrm{~mm}$ camera. The filter in front of the camera was a yellow glass-absorption filter that had been selected to block the reflected lamplight. An APD investigator took multiple camera exposures of each sample with direct and diffuse illumination in a dark room. The photographs with best contrast were scanned into a computer file and are displayed on the left-hand side of Figure 7.

The right-hand side of Figure 7 displays the images captured by CLU for the same control samples. To demonstrate the advantages of our system, we imaged these samples shortly after the APD photographs were taken. We used a $70 \mathrm{~nm}$ bandpass filter centered at $455 \mathrm{~nm}$ in front of the lamp and a $515 \mathrm{~nm}$ long-pass filter in front of the camera. The intensifier gain was set to exercise the full dynamic range of the camera. The resulting image was recorded on $8 \mathrm{~mm}$ videotape and later digitized to a computer file. The contrast for the APD and CLU images was maximized by expanding the gray-scale histogram to use the full range of brightness values.

A comparison of the APD photographs and CLU images shown in Figure 7 illustrates the improved sensitivity and specificity achieved by CLU, as well as the ambient-light rejection. All of the APD photographs were taken in a dark room, while the CLU images of samples \#1 through \#4 were taken in a fully lit room. Because the ratio of semen-to-fabric brightness is higher in the CLU images, the specificity has been improved. In samples \#5 and \#6, the stains were neither visible to the conventional technique nor were they visible to CLU in a lit room. In a dark room, however, the camera gain on CLU was increased to capture the stain images shown in samples \#5 and \#6. The fact that CLU can capture images of stains that are invisible to the conventional technique indicates improved sensitivity that will allow investigators to locate semen evidence that is currently not detected. The fact that stains on dark materials are visible to CLU only in darkened rooms emphasizes the importance of training investigators about the types of backgrounds that are difficult for locating stains. Fortunately, the difficult backgrounds will be the same for CLU as they are for existing alternative light sources. 


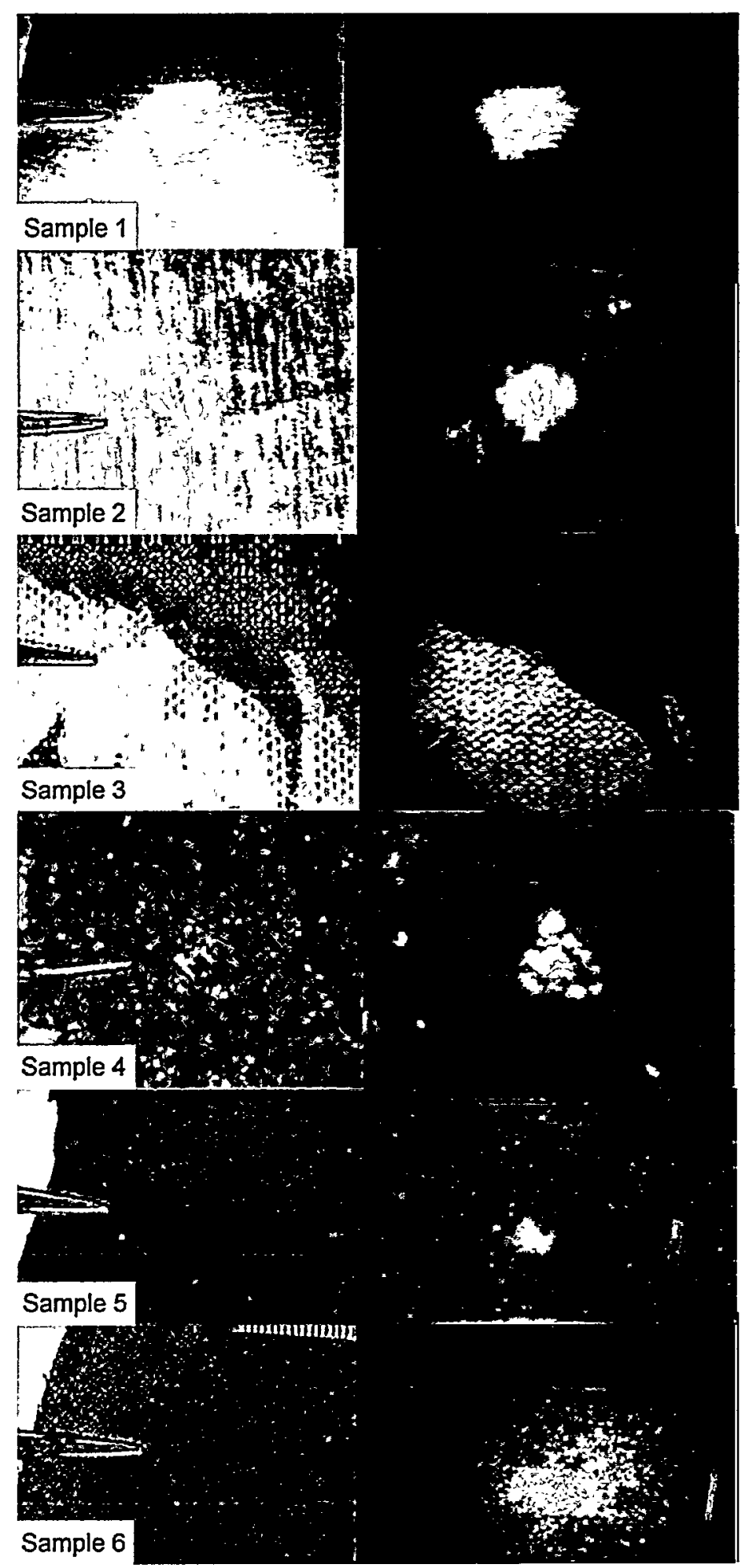

Figure 7. Semen-Stain Images of the APD Control Samples Taken by the Conventional Technique (Left) and Then by CLU (Right). 


\section{Exploration of Contrast-Improvement Methods with CLU}

To detect semen, alternative light sources currently use blue excitation light in the region of $450 \mathrm{~nm}$. In general, the intensity of biological fluorescence increases as the excitation wavelength moves toward the ultraviolet (UV). One possible method of increasing the semen fluorescence against the background, and thus making the stain more visible, is to look for an excitation peak toward the UV. To explore this method, we took images with CLU of the APD control samples (in Table 2) using the filter combinations shown in Table 3.

Table 3. Visible and UV Filter Wavelengths

\begin{tabular}{|c|c|c|}
\hline $\begin{array}{c}\text { Image } \\
\text { Identifier }\end{array}$ & Excitation & Emission \\
\hline L1 & $70 \mathrm{~nm}$ BP @ 455 nm & $515 \mathrm{~nm} \mathrm{LWP}$ \\
\hline $\mathrm{L} 2$ & $80 \mathrm{~nm}$ BP @ 330 nm & $400 \mathrm{~nm}$ LWP \\
\hline L3 & $20 \mathrm{~nm}$ BP @ 280 nm & $325 \mathrm{n} \mathrm{m} \mathrm{LWP}$ \\
\hline
\end{tabular}

In Table 3, BP in the excitation column refers to the limited passband given by the first number centered at the wavelength given by the second number. For example, "70 nm BP @ $455 \mathrm{~nm}$ " describes a full-width half-maximum passband of $70 \mathrm{~nm}$ with peak transmission centered at $455 \mathrm{~nm}$. In the Emission column, LWP refers to a long-wave pass filter that passes wavelengths above the number listed and attenuates lower wavelengths by a factor of $10^{4}$. The L2 and L3 UV wavelengths were selected to match known excitation and emission peaks for amino acids, common biological fluorophores. Figure 8 presents the images taken with CLU of control sample \#1 (from Table 2) with the various filter combinations in Table 3 . These images show that the shorter UV wavelengths actually decrease the semen intensity relative to the background. A similar decrease in contrast was seen for all six control samples at the L2 and L3 wavelengths. These results demonstrate that using UV light for semen imaging is actually a disadvantage rather than an improvement.
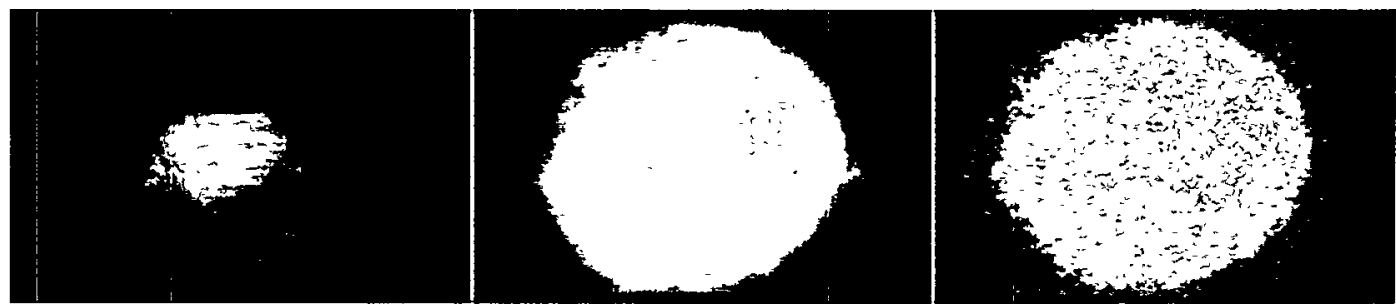

Figure 8. CLU Images of a Semen Stain on $100 \%$ Cotton Fabric (APD Control Sample \#1) Taken with Different Filter Combinations. 
Another possible method for improving the fluorescence is to limit the passband of the emission filter in front of the camera. The purpose of limiting the passband is to reduce the background light detected relative to the semen fluorescence. The background light includes room light, reflected excitation light, and substrate fluorescence. To determine the benefit of reducing the passband, we added short-wave pass (SWP) filters to the long-wave pass filters in front of the camera. Table 4 lists the filter combinations that were used with CLU to limit the emission passband. The images in Figure 9 of a semen stain on upholstery (APD control sample \#3) show a moderate advantage to the band-limiting filters. Measurements of the semen and background brightness indicate that limiting the passband to a $45 \mathrm{~nm}$ width with the 560 SWP filter improves the contrast by a factor of 1.4. Hence, the benefit of the additional filter is marginal. The SWP filter should be included in future evaluations of difficult backgrounds not considered here.

Table 4. Filters Used to Limit the Wavelengths Seen by the Camera

\begin{tabular}{|c|l|l|}
\hline $\begin{array}{c}\text { Image } \\
\text { Identifier }\end{array}$ & \multicolumn{1}{|c|}{ Excitation } & \multicolumn{1}{c|}{ Emission } \\
\hline No F & $70 \mathrm{~nm}$ BP @ 455 nm & $515 \mathrm{~nm}$ LWP \\
\hline F1 & $70 \mathrm{~nm}$ BP @ 455 nm & $515 \mathrm{~nm}$ LWP \& 560 nm SWP \\
\hline F2 & $70 \mathrm{~nm}$ BP @ 455 nm & $515 \mathrm{~nm}$ LWP \& 600 nm SWP \\
\hline
\end{tabular}
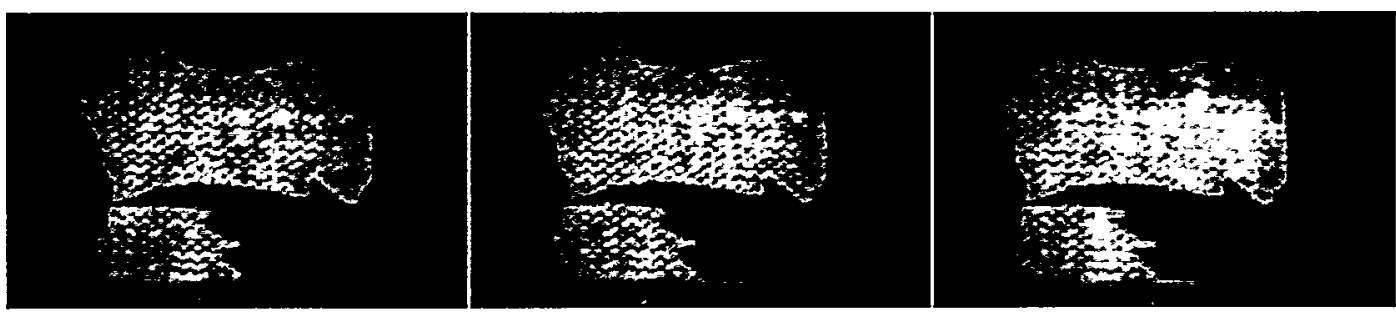

Figure 9. CLU Images of a Semen Stain on Upholstery Fabric (APD Control Sample \#3) with Various Emission Passbands.

\section{Time-Series Test}

Previous research has noted that fluorescent stains can fade over time in some samples [26]. We monitored the intensity of the APD-provided control samples over several months to see whether the intensity faded with time. As shown in Figure 10, the fluorescent images captured by CLU of control sample \#1 at the start of the evaluation and after two months demonstrated only a minor decrease in intensity. The sample was exposed to ambient room temperature in a laboratory. The other samples showed similar stability. 


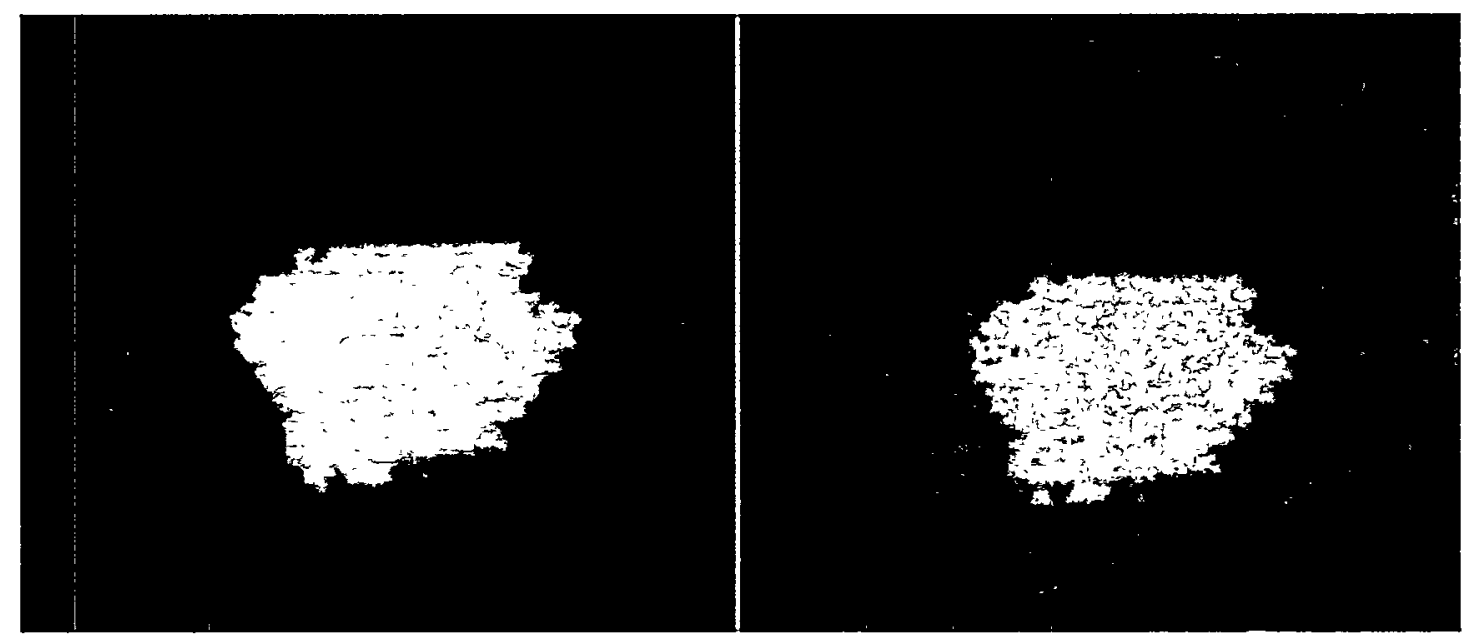

Figure 10. CLU Images of Fluorescence of the Same Semen Stain (APD Control Sample \#1) over a Two-Month Period.

\section{Test on Living Human Skin}

In the investigation of a sexual assault, the primary source of semen evidence is often the victim. Thus, another important application of fluorescence imaging is being able to find semen on human skin. Because skin tends to fluoresce strongly, the semen fluorescence is typically hard to see. As part of the APD evaluation, a serologist volunteered to place a semen stain on her skin. The resulting image in Figure 11 of the dried stain on her skin demonstrates the contrast captured by CLU. Although we did not document semen on human skin with an alternative light source or Woods lamp, investigators stated that the image in Figure 11 is better than images produced by those conventional techniques.

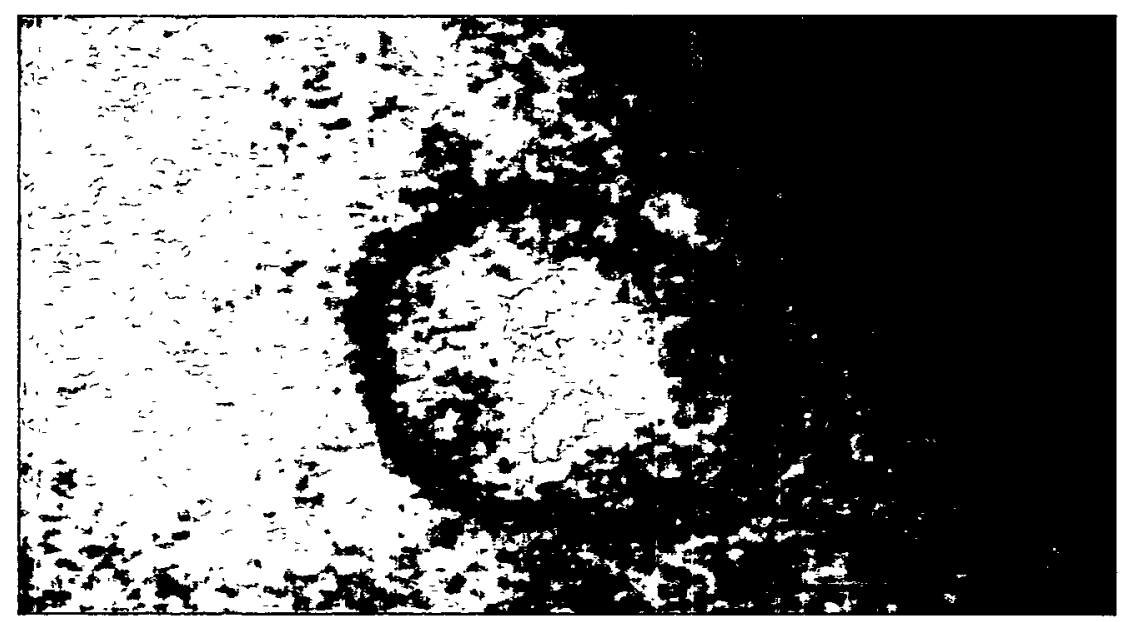

Figure 11. CLU Image of Semen Fluorescence on Living Human Skin. 


\section{Field Tests}

As part of their evaluation of the system, APD allowed us to use CLU in a murder investigation. The victim was found several days after her death and was beginning to decompose. Our initial effort involved screening the corpse in the morgue for possible semen stains to determine whether a sexual assault had occurred. With the room lights on, we located three possible stains on the pelvis area and upper thigh. Then, with the room lights off, APD investigators attempted to view the same possible stains with the alternative light source, but the reflection from fluids on the skin surface overwhelmed any fluorescence. An APD serologist subsequently sampled the stains located by CLU, and her analysis revealed sperm cells in one of the samples. The number of sperm cells found was extremely small, indicating a high sensitivity for the system. Unfortunately, we are unable to show images of the stains in this report because the videotape is being held for evidence.

We also screened the site of the murder for possible semen stains on the carpet and mattress. Again, we imaged the fluorescence with the room lights on and blinds open. Several possible stains were located on the white carpet but field-tested negative for semen.

\section{Reflectance Imaging of Bloodstains}

CLU's ability to image bloodstains was evaluated in tests with the APD Crime Lab. To increase the contrast of the bloodstains, we used reflected light, rather than fluorescent light, and maximized the absorption contrast of blood with a narrow $10 \mathrm{~nm}$ bandpass filter in front of the camera. The peak transmission of the filter was centered at $415 \mathrm{~nm}$, which is the dominant absorption peak of dried blood. The net effect of this configuration is that the area with blood appears dark against the background. For eye protection, we placed a long-pass filter in front of the lamp to block the UV light emitted by CLU.

\section{Test on Dark Background}

This test evaluated CLU's ability to image dried blood on a dark background. APD investigators had dripped blood on a piece of bark and allowed it to dry. The photograph on the left side of Figure 12 demonstrates the difficulty in locating the bloodstains with the naked eye. The contrast of the stains against the bark is improved in the CLU image on the right side of the figure, allowing the observer to determine that there are two small blood spots within the area of the red box. 


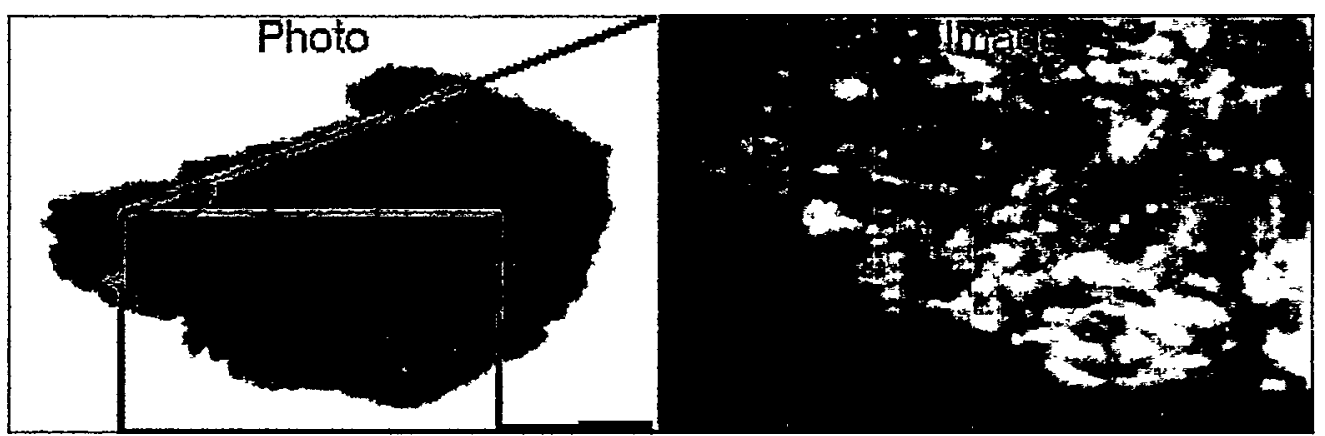

Figure 12. Photograph (Left) and CLU Image (Right) of Dried Bloodstains on a Piece of Tree Bark.

\section{Test of Cleaned-Up Evidence}

CLU was also tested for its ability to locate trace amounts of cleaned-up blood. APD investigators allowed several blood drops to dry on the rock pictured in Figure 13. Then, simulating an activity of those who want to conceal a crime, the investigators attempted to clean the dried blood off with water and abrasion. The stains are somewhat visible in the photograph; however, we expect that the stains would be quite difficult to see when a large area was being searched. Again, the improved contrast obtained with CLU allows the user to locate the spots on the rock. While we do not expect the reflectance-imaging technique to have the same sensitivity as luminol, we believe that the simplicity of this technique would result in wider usage than luminol and in the detection of many residual bloodstains.

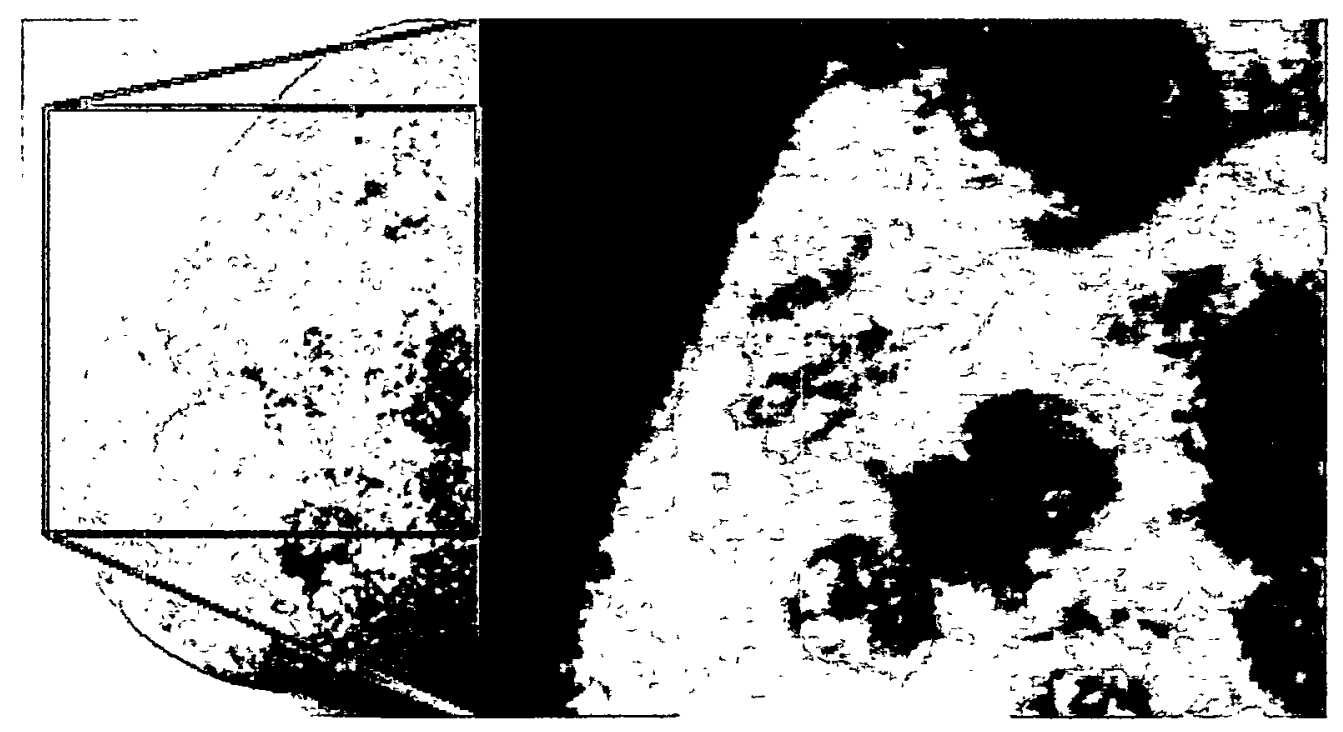

Figure 13. Photograph (Left) and CLU Image (Right) of Cleaned Bloodstains on a Rock. 


\section{Imaging of Fingerprints}

We dedicated a large effort to developing a technique that can image untreated fingerprints. Ideally, this technique will speed up the search process, enhance the quality of evidence collected, and eliminate much of the mess and exposure to chemicals. In practice, the technique should allow the user to search a field-of-view that is one foot in diameter. If a suspected print is located in the large field-of-view, the user should be able to zoom onto the print and capture the ridge detail needed for identification. In addition to meeting the technology needs of investigators, the everyday need to find fingerprints creates a large potential market for the end product.

\section{Native Fluorescence}

Based on previous work describing the imaging of untreated fingerprints with their natural fluorescence, we attempted to image the fingerprint fluorescence using CLU. Unfortunately, we were unable to image fingerprints with fluorescence at the greatest camera sensitivity. To confirm that the problem was a lack of fluorescence rather than equipment error, we measured the fluorescence spectra from fingerprints using a highperformance system at Sandia. That system uses a laser tunable over the UV and blue wavelengths for a light source, and an intensified CCD sensor for a detector. Together, the laser and CCD sensor provide one of the most sensitive fluorescence detection systems available today. The plots of the fingerprint spectra in Figure 14 show the two fluorescence peaks. These plots represent the fluorescence strength measured from a fingerprint at various wavelengths, or colors of light. The plots confirm that fingerprints fluoresce naturally at two UV wavelength bands. However, the plots also indicate that the natural fluorescence is very weak and below the detection limit of CLU.
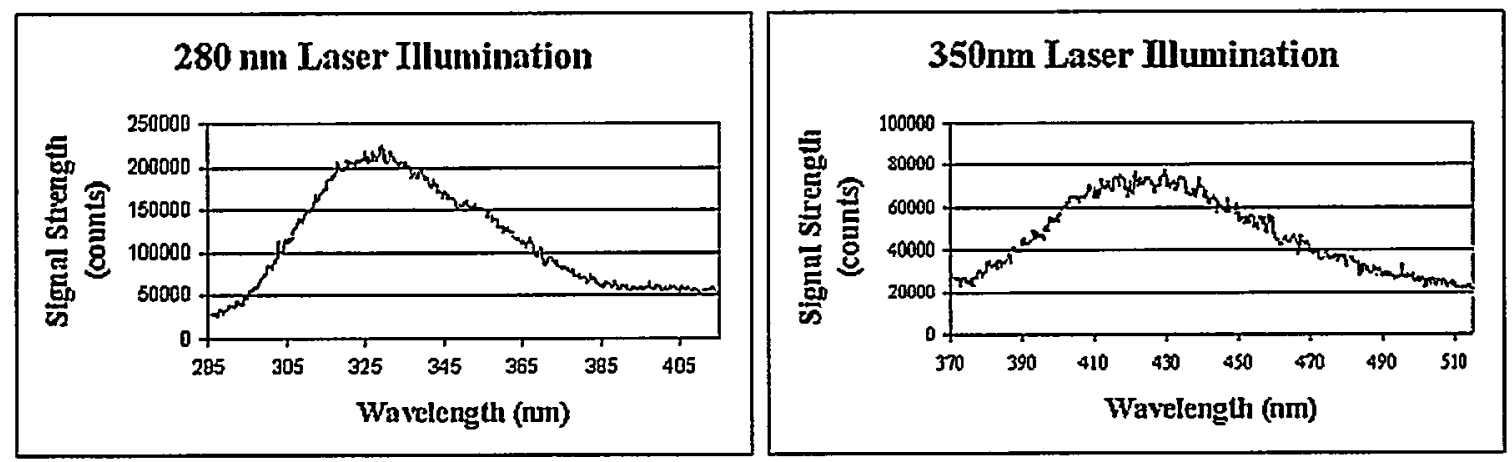

Figure 14. Fluorescence Spectra from Untreated Fingerprints.

Our measurements of fingerprint fluorescence strength demonstrated that the imaging of native fingerprint fluorescence is not a practical method for field investigations. Most of the fingerprint spectra measured were heavy in sebaceous content. The fluorescence from some fingerprints low in sebaceous content either was below the detection limit of the high-performance system or did not exist. Our signal-strength measurements suggest 
that an effective imaging system would require a UV laser for illumination. However, the cost, size, and safety concerns of a UV laser make it impractical for field use. Another option for imaging native fingerprint fluorescence would be to use a high-power UV lamp for illumination and an ultra-low-noise scientific-grade camera, similar to the one used for the above measurements, for detection. This camera could image the fluorescence created by the lamp over tens to hundreds of seconds to build up an image of the fingerprint. However, the cost of the camera $(\$ 30,000-\$ 40,000)$ and the time the camera would have to be held motionless make this approach unattractive for field applications. In both approaches discussed above, fluorescence imaging at this low level could not be done in ambient light, thereby limiting its application.

\section{Reflectance Imaging}

While the lack of fingerprint fluorescence was disappointing, an alternate method for imaging fingerprints was discovered. Using fluorescence imaging, CLU was showing black images even with the camera set at the maximum sensitivity. To verify that the camera was working, we removed the filter in front of the camera and were surprised to see a bright image of the untreated fingerprint against a dark background. The detail in that initial image was sufficient to see the individual ridges of the fingerprint. Being able to visualize the ridges and to locate the identifying points of the fingerprint without treatment met the goals of our research. Without the camera filter, CLU uses reflected light to image fingerprints, just as human vision uses reflected light to see the world around us. Figure 15 illustrates the quality of a print image that can be obtained with reflected light.

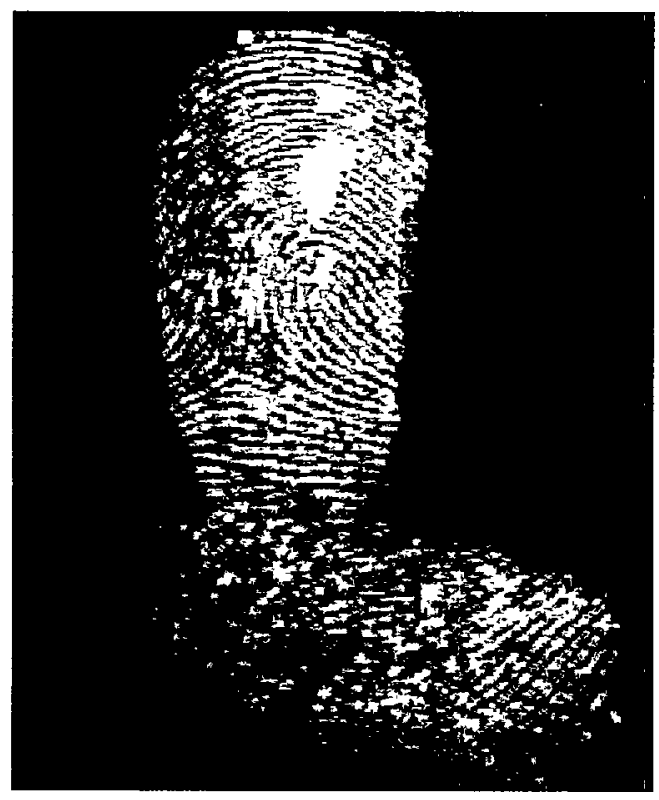

Figure 15. Reflected White-Light Image of a Fingerprint on a Glass Slide. 
Based upon this initial image, we pursued reflectance imaging as a possible technique to rapidly visualize untreated fingerprints with a minimum of equipment. To evaluate the technique, we used CLU to image fingerprints on various substrates under different lighting and observation conditions. Most of the tests were done in conjunction with personnel from the APD Crime Lab. The samples were selected to represent a range of surface conditions and materials that might be found in a crime scene. Although it is not possible to demonstrate the results on all surfaces given the number of possibilities, it may be possible to extrapolate the results provided here to other surfaces of interest. All of the images shown below represent untreated fingerprints. In the APD tests, each of the sample objects was placed in front of the camera and illuminated by the light guide that was mounted at the side of the camera. The resulting fingerprint image was recorded on videotape and later digitized to a computer file. The gray-level histogram of the resulting image was expanded to use the full range of values and to maximize contrast of the image.

The glass surface of Figure 15 (shown above) is ideal for the reflectance technique. The technique works quickly and accurately on any surface that is flat, smooth, and transparent. The print images from these surfaces are clear and provide sufficient quality for entry into AFIS. Although this surface description may seem like a limited area of application, the flat, smooth, and transparent surfaces are encountered in crime scenes (e.g., the window through which a burglar entered, the window and rearview mirror of a victim's car, and a compact-disc case on a ransacked desk). We have had similar success imaging fingerprints on plastic bags when the bags can be stretched tight to form a flat surface.

Obviously, many of the surfaces in a crime scene are not transparent. The difficulty of imaging a print from a surface can be estimated from the print's variation in color and from its surface roughness or texture. APD investigators selected the plastic ruler in the upper-left corner of Figure 16 for its variegated color and slight surface texture. We used reflected light to capture both of the fingerprint images shown in Figure 16 from the ruler. An APD print examiner thought that both images contained enough ridge detail to identify the prints on a comparison basis (i.e., with the actual fingerprints of a known suspect) but that the images contained too much background information to be entered into AFIS. 


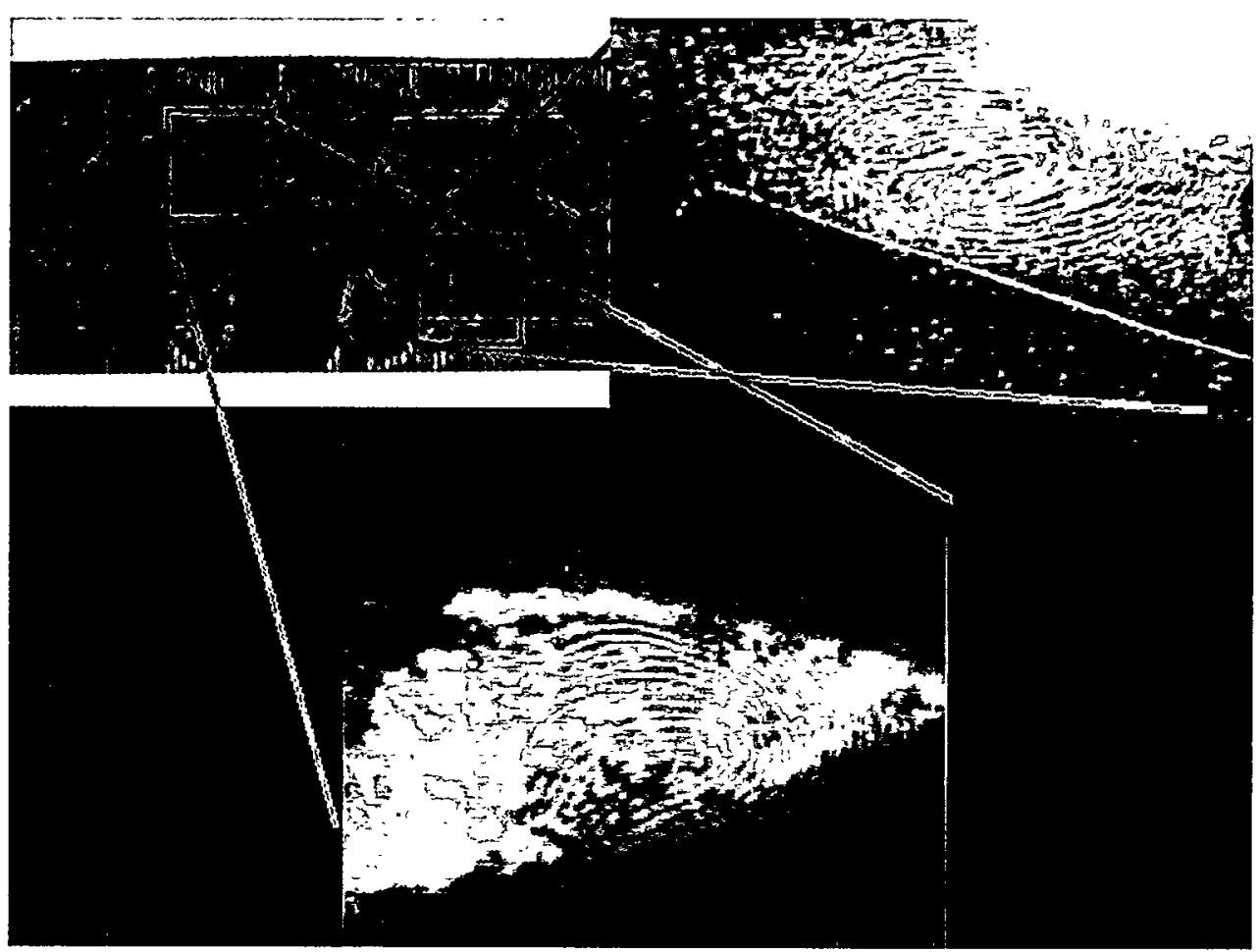

Figure 16. Photo of Plastic Ruler and Images of Two Fingerprints Captured from Ruler.

The fingerprint images at the top and bottom of Figure 17 were captured from each side of the pistol. Enough ridge detail is captured with diffuse imaging to identify the print on comparison; however, a large background is created by the tooling marks on the slide.

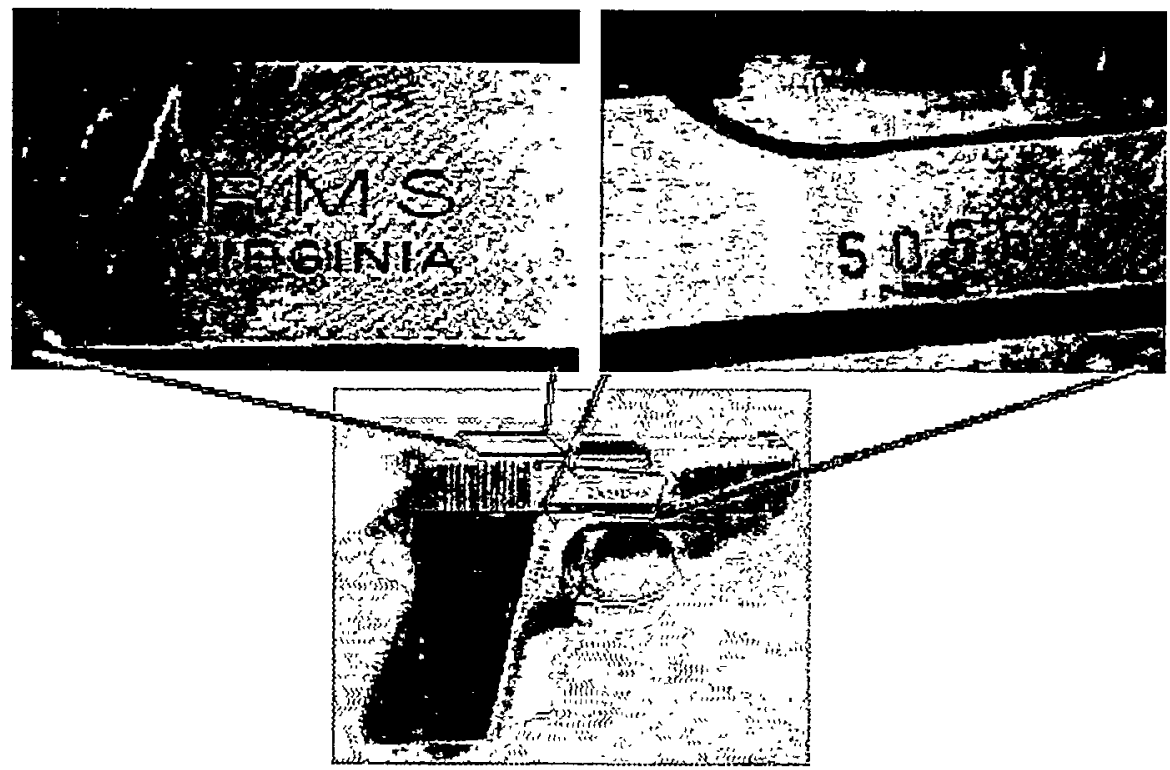

Figure 17. Fingerprint Images Captured from Near and Far Side of Pictured Pistol. 
Figure 18 shows the fingerprint image captured from a plastic security badge. APD investigators chose the badge for its holographic texture and variegated color pattern. Unfortunately, no photo was taken to show the print's appearance. An APD examiner judged that the print quality would be acceptable to AFIS. Before the print could be submitted to AFIS, however, its contrast would need to be reversed so that the ridges are black against a white background and the magnification is scaled to 1:1 with the original print. The fingerprint shown in Figure 18 differs from the previous figures in that it is heavy in sebaceous oil content. With increased oil content, the quality of the reflected light fingerprint image improves. The majority of fingerprints imaged here were placed by an APD investigator who is known for leaving light, nonoily fingerprints.

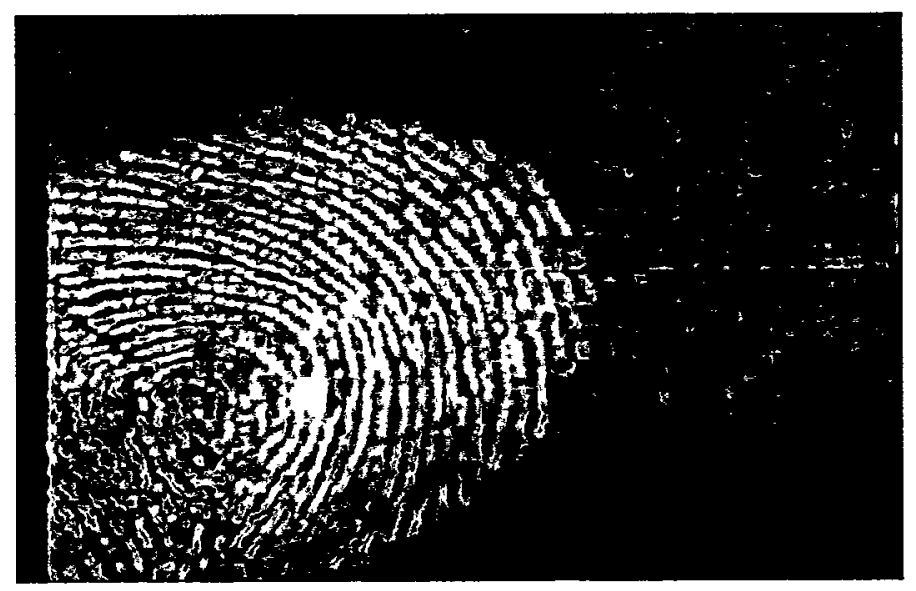

Figure 18. Fingerprint Image Captured from Plastic Security Badge.

APD investigators suspected that the reflected light technique might not work on bright, highly reflective surfaces so they picked the white plastic on the left of Figure 19 for a sample surface. The fingerprint image captured from the area of the red box shows enough ridge detail to identify the print on a comparison basis. This fingerprint was light in oil content.

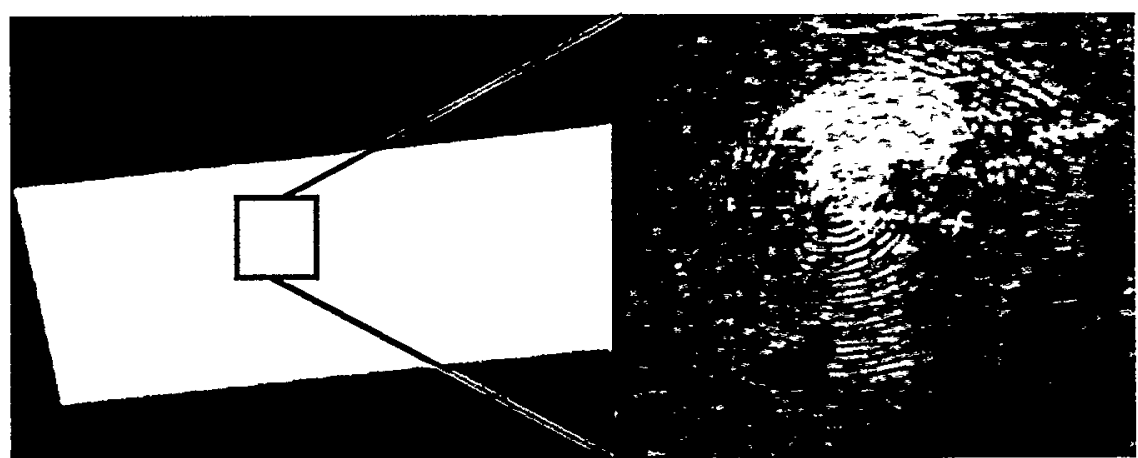

Figure 19. Photo of White Plastic Sample and the Fingerprint Image Captured from the Sample's Surface. 
Formica, used as the sample in Figure 20, is a common household surface. The fingerprint image on the right of Figure 20 was captured from the area of the red box by reflectance. The image quality was judged good for a comparison with a known suspect's fingerprints but marginal for submission to AFIS.

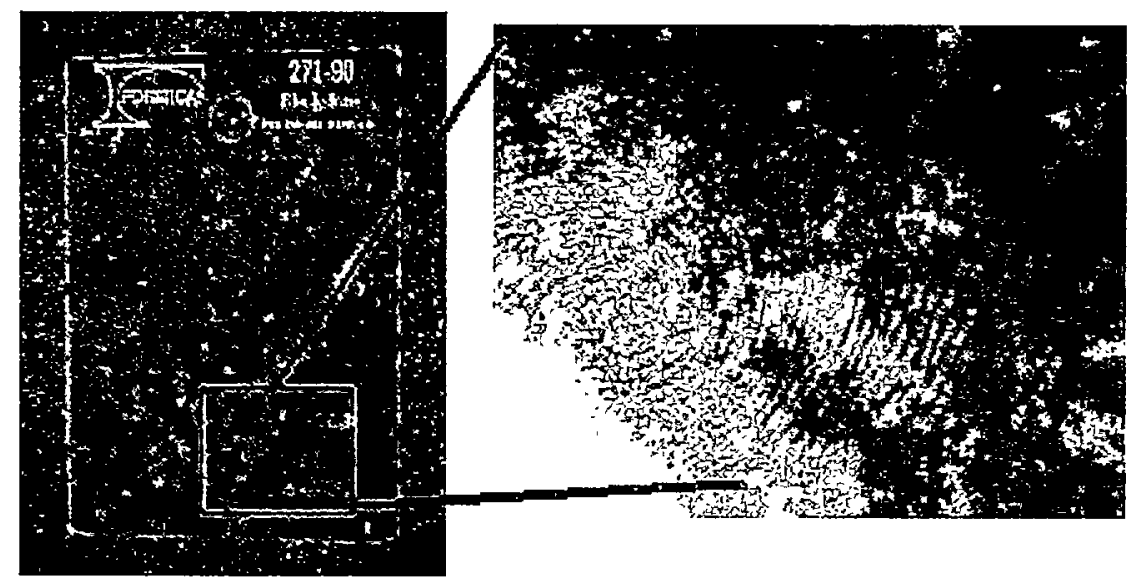

Figure 20. Photo of Formica Sample and the Fingerprint Image Captured from the Sample's Surface.

Some common surfaces are manufactured with a roughness to hide fingerprints. The Formica sample on the left of Figure 21 was selected as an example of a rough surface. We captured the fingerprint image from the area of the red box by reflectance. The fingerprint image was judged acceptable for comparison with a known suspect's fingerprints. A heavy fingerprint, though, was required to produce an acceptable image on this rough surface. A fingerprint lighter in oil content was visible but appeared to have discontinuities in the ridges at the lows, or valleys, of the surface texture. We do not know whether the inability to see light fingerprints on rough surfaces is a limitation of the reflectance technique or the physical reality that no fingerprint material is left in these areas.

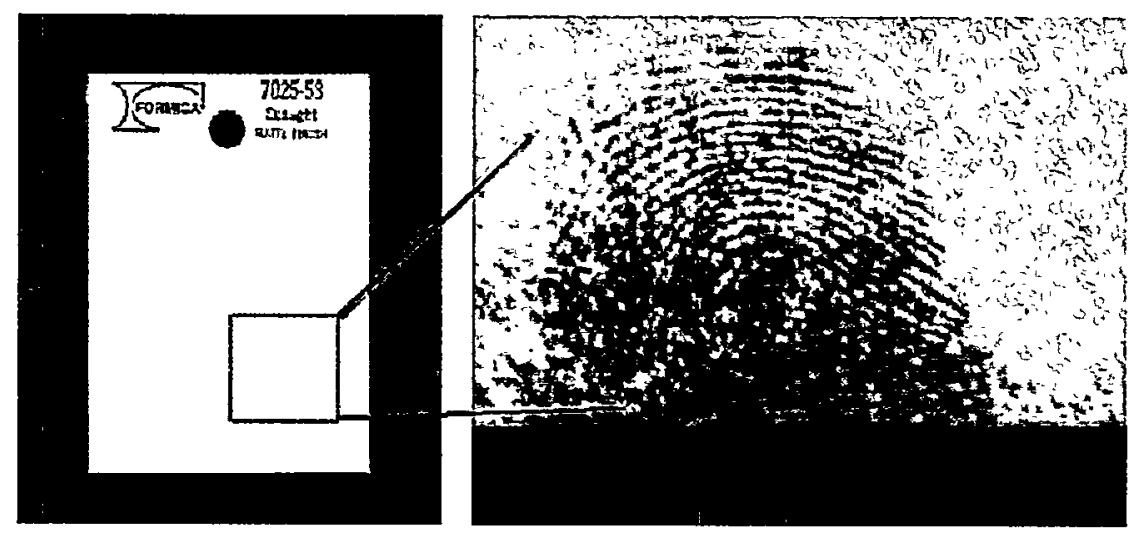

Figure 21. Photo of Rough Formica Sample and the Fingerprint Image Captured from the Sample's Surface. 
To illustrate the results from additional surface textures, we captured the fingerprint images in the areas of the red boxes in Figure 22 from a computer floppy disk. Both fingerprints were considered to be light in oil content. The degree of roughness in the plastic was less than the Formica of Figure 21, which allowed us to see the light fingerprint.

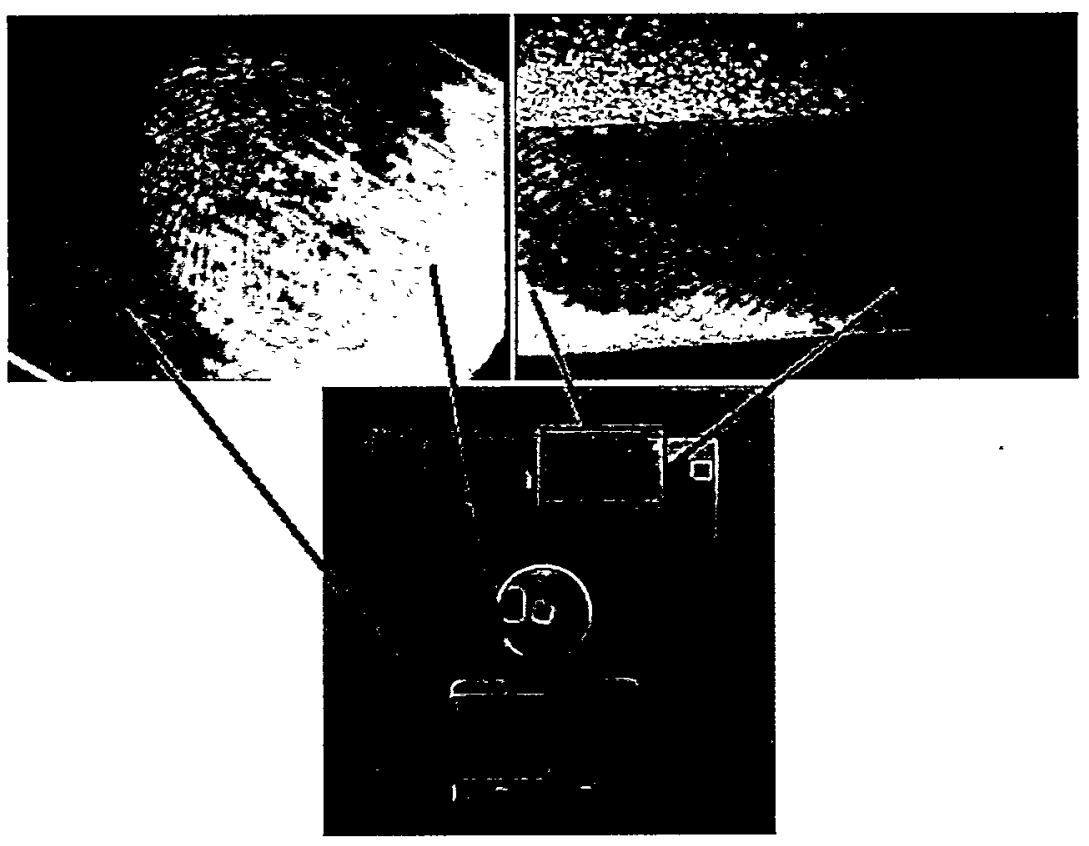

Figure 22: Photo of Computer Floppy and the Fingerprint Images Captured from the Area of the Red Boxes.

Fingerprints at a crime scene are often left on curved surfaces such as cans, bottles, bullet casings, gun barrels, and some curved knife blades. Currently, the reflectance technique is limited to capturing a portion of the fingerprint from a curved surface. In the image from a curved knife blade (see Figure 23), only the edges of a print are visible around a bright reflectance at the center of the blade.

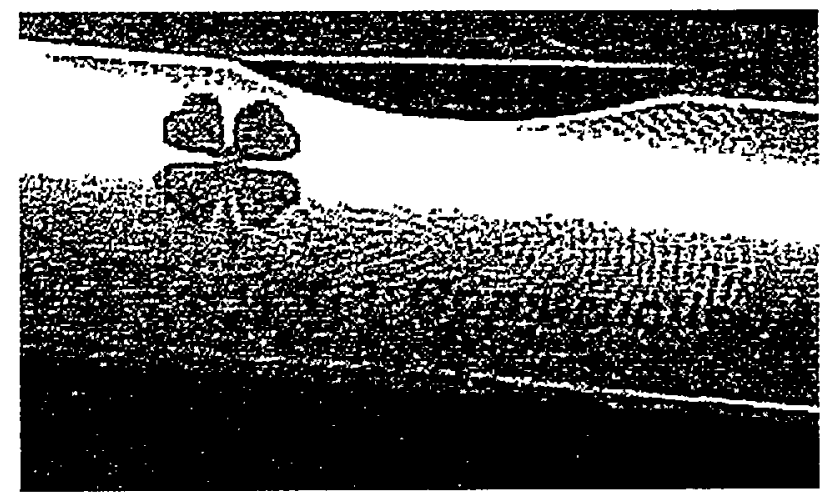

Figure 23. Reflectance Image of a Fingerprint on a Curved Knife Blade. 


\section{Imaging of Gunpowder Residue}

To improve the technique for screening an individual for firing a gun, we hoped to find an absorption or reflectance peak for gunshot residue (GSR) that would provide contrast against a skin or cloth background. If it is possible to visualize GSR, we imagined that it would be possible to differentiate a shooter from an adjacent person by the distribution of the GSR on the hand. To test this hypothesis, we fired a revolver through a 100-percent cotton-cloth sample several times. A second piece of cotton cloth wrapped around the rear of the gun captured the GSR directed at the shooter. The front and rear samples were analyzed for transmission and reflectance spectra from the UV through the visible to the infrared (IR) region. Unfortunately, the spectra, shown in Figure 24 do not contain any spectral features in the UV to visible region that CLU could use to differentiate the GSR from the background. There is an absorption peak in the IR region at the $9 \mu \mathrm{m}$ wavelength. However, CLU cannot operate at these wavelengths and we did not have access to an IR camera to evaluate the GSR imaging.

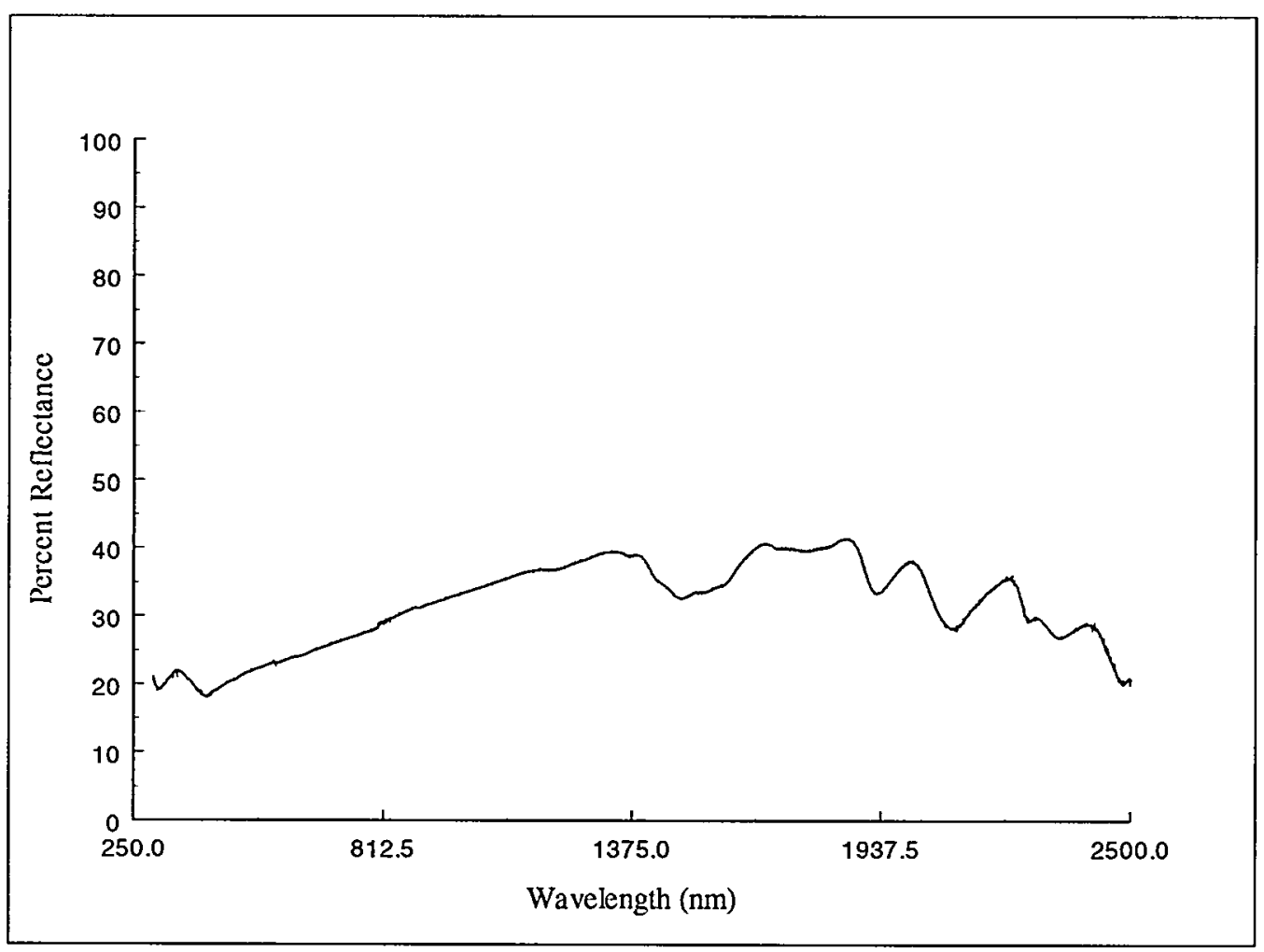

Figure 24. Transmission and Reflectance Spectra of Cotton Containing GSR Relative to New Cloth. 


\section{Discussion}

This section evaluates the imaging capabilities of CLU to meet the project goals, points out areas of system improvement, and describes our efforts to commercialize the technology.

\section{Fluorescence Imaging}

With CLU, Sandia has met the project goals of rejecting ambient light and improving sensitivity (and specificity) to the target substance. Compared to conventional methods of fluorescence detection, CLU has demonstrated the abilities to reject ambient light by a factor of approximately 100 and to increase sensitivity by an estimated factor of 5 .

Sandia investigated the use of UV light in CLU to detect fluorescing evidence, based on promising research results described in the Background section. Several system components (the lamp, image intensifier, and camera lenses) were selected, in fact, because of their ability to work in the UV region of the optical spectrum. During evaluation tests of the system with APD, however, we found that using UV wavelengths did not enhance the results. For example, we observed that while semen stains fluoresce more at the shorter UV wavelengths, so does the background substrate. Our finding that a UV capability offers no advantage is significant because it reduces the cost of the system by approximately one half.

Sandia also investigated the use of CLU to detect inherent fluorescence from fingerprints, based on experimental work described in the Background section. Our experimental results showed that while the technology is feasible, it is not practical as a search application in the field. For example, a system capable of visualizing inherent fluorescence would require an intensified camera whose signal would have to be integrated for several minutes. Even then, the system would not be able to detect inherent fluorescence in any kind of ambient light. The equipment would also be expensive (approximately $\$ 100,000$ ) and bulky (approximately 150 pounds).

\section{Reflectance Imaging}

During our experimental work with inherent fluorescence, we discovered that by taking the filter off the front of the camera we were able to observe untreated fingerprints fairly well just from reflected light. This finding led us to further explore the potential of direct reflectance as a technique for meeting the enhanced project goal of visualizing multiple types of evidence. Reflectance is the basis of human vision and produces a strong optical signal. As results from evaluation tests with APD demonstrated, we were able to use reflectance imaging to reveal blood evidence, screen and record fingerprint evidence on various flat surfaces, and image partial fingerprints off curved surfaces. A major difficulty in using reflectance imaging with CLU is the time required to capture a fingerprint. It currently takes from 2 to 40 minutes to capture a fingerprint. Ongoing 
research should reduce the time to 30 seconds and reduce the amount of background signal from the surface.

\section{System Development}

Although CLU met the project goals, it has not been optimized for use in the field. The system is approximately 19 inches tall, 24 inches wide, and 25 inches deep; it weighs about 95 pounds. The system's bulk results from our attempt during the design phase to develop a research instrument that was sensitive to the range of conditions, types of evidence, and types of backgrounds encountered by investigators at crime scenes. In assembling this system, we used discrete commercial products that are intended for research. Many of these products/components are bulky and expensive. The cost in components to assemble the prototype was approximately $\$ 30,000$.

The current system can be optimized for use in the field through reductions in complexity and size, which will significantly reduce the cost. The system is composed of individual controllers (for lamp, camera, and video integration) that contain redundant power supplies and features not used in the forensic applications. These controllers could be designed as plug-in modules that use a single power supply, which would result in a lighter weight, more compact, and efficient system. We anticipate that the size could be reduced by a factor of four to about 25 pounds, roughly the size of a large briefcase, and the weight by a factor of three. Additional savings in size can be achieved by using smaller components for the lamp and camera. Finally, as noted in the discussion of fluorescence imaging, by eliminating the UV capability, we can reduce the overall cost of the system by a factor of two.

Our discovery of CLU's potential to use reflectance imaging led us to conclude that the prospects for maximizing the system's usefulness to law enforcement would best be served by two instruments: a full-blown optimized version of the system and a hand-held derivative version of the system, as described below.

Full-blown System: This instrument would perform both fluorescence and reflectance imaging. It could be used by specialists in crime-scene investigations. Every crime lab could afford one or two of these units at a cost of approximately $\$ 10,000$ to $\$ 15,000$ per unit.

Hand-held System: This instrument would perform only reflectance imaging to capture fingerprints and blood evidence. Because reflectance is such a strong optical signal, there is no need for the capability of rejecting ambient light. Instead, the system illuminator overpowers the ambient light. The components of the hand-held system would include a customized camcorder with illuminator optics and filters at a cost of approximately $\$ 3,000$ per unit. Individual police departments would be able to purchase several of these units. For the first time, the cost and size of advanced technology would allow deployment of multiple units in cruisers, rather than one copy in the lab or major crime van. In effect, the hand-held system would enable deployment of the technology to the scenes of lesser crimes, which occur in greater 
numbers. It should be noted that improvements in the reflectance imaging capability are required before this product could be successfully commercialized.

During this NIJ project, Sandia did put together a rough version of the hand-held system. It's performance in reflectance imaging, however, did not mirror that of CLU's. We identified the need for improved optics as the major problem and submitted a proposal to NIJ to further research and develop the approach.

\section{Technology Transfer}

Our efforts to commercialize the technology began with a suggestion by NIJ to find a corporate partner. We placed an ad in the Commerce Business Daily and received four responses. Two companies followed through on the paperwork but only one was capable of bringing the concept to market: Molecular Technologies, Inc. (MTI).

In September 1997, Sandia entered into a Cooperative Research and Development Agreement (CRADA) with MTI. MTI's primary role was to conduct the market research. Currently, with our assistance, MTI is searching for a potential manufacturer. Several parties have expressed interest in further developing the technology. One company is pursuing venture capital and has declared its intent to transfer the technology and manufacture it.

It is also important to note that the imaging capabilities demonstrated in CLU can be used for applications beyond the domain of crime-scene investigations and labs. One potential application is in the inspection of visual coatings, such as wire harnesses in aircraft that would fluoresce when the insulation was wearing out or paint on petroleum tanks that would fluoresce when parts were starting to rust. Another potential application is in the visualization of fluorescent tracers, whereby the origin of explosives could be traced, by specific wavelengths, to the manufacturer of the devices and point-of-sale outlets. A third possible application covers the system's use as a diagnostic tool to inspect biological contaminants in surgical equipment. During this NIJ project, Sandia has received inquiries from manufacturers about these various applications.

Sandia has documented technical advances and is pursuing patent applications on the concepts of ambient-light rejection and reflectance imaging developed in this NIJ project.

\section{Conclusions}

Throughout this NIJ project, Sandia extensively researched and observed the currently used evidence-gathering practices of local law enforcement. The design of CLU was, therefore, based on the strengths and limitations of these practices and the needs for new technologies, as indicated by forensic researchers and as voiced by our technical advisors and system evaluators-investigators in the APD Crime Lab. We selected system components for CLU primarily to meet the technical project goals of rejecting ambient light, increasing sensitivity and specificity of fluorescing evidence to the target substance, and visualizing multiple types of evidence. At the same time, however, we 
strove to develop a system that could be used efficiently in the field, that was affordable to local police departments, and that was safe to use. Safety was a primary concern, given that some of the current evidence-gathering practices pose risks to investigators, crime victims, and to the evidence itself.

In its first incarnation as a prototype imaging system, CLU has successfully achieved the technical project goals and is able to visualize evidence in a manner that is safe for both personnel and evidence alike. Employing both fluorescence and reflectance imaging capabilities, CLU exceeds the performance of current technologies and methods available to local law enforcement. Investigators can now observe fluorescing evidence such as semen stains in daylight hours, eliminating the need to black out windows as is often required. CLU also demonstrates the potential through reflectance imaging to locate blood evidence on dark surfaces, and untreated fingerprints on transparent, opaque, and even multicolored surfaces. The reflectance-imaging capability, which uses the visible rather than UV spectrum, is not currently available in any commercial product used by local law enforcement. We believe that the use of reflectance imaging in the visible spectrum to image untreated fingerprints can open a whole new regime in forensic technology. Through an analog-video-recording feature, CLU allows investigators to view the entire search process in real time and submit the recorded documentation as evidence in a courtroom.

Further development is needed, however, before the imaging capabilities in CLU can be successfully commercialized for use in crime-scene investigations. Given time and cost constraints in this project, the system was not optimized for field performance and requires reductions in complexity and size to meet the portability and affordability requirements of local law enforcement. Sandia has proposed that the prototype be further developed as two separate instruments, a full-blown optimized version of CLU and a derivative hand-held system. In this report, we have discussed the improvements necessary to bring these products to market and our efforts thus far to commercialize them. Importantly, users of both instruments (and bystanders) would not have to be concerned with their personal safety since no equipment would be employed that poses any risks to the eyes or skin. And the evidence would be obtained in a noninvasive fashion that leaves no residue for crime victims to clean and that leaves the evidence intact for subsequent analysis. The hand-held instrument, in particular, holds considerable promise as a cost-effective and easy-to-use tool in field investigations for imaging blood and untreated fingerprint evidence. This small instrument would enable the collection of evidence that may be overlooked at lesser crime scenes.

\section{References}

1. Bureau of Justice Statistics, U.S. Department of Justice, "Crime and Victims Statistics," Available: http://www.ojp.usdoj.gov/bjs

2. Bureau of Justice Statistics, U.S. Department of Justice, "Criminal Victimization, General," Available: http://www.ojp.usdoj.gov/bjs 
3. Schiro, G., "Collection and Preservation of Blood Evidence from Crime Scenes," Available: http://police2.ucr.edu/blood1.html

4. Macko, S., "The Advancement of Fingerprint Technology," Available: http://www.emergency.com/fngttech.htm

5. Sirchie Fingerprint Laboratories, Product Literature, Company Address: 100 Hunter Place, Youngsville NC, 27596.

6. James, J. D., Pounds, A., and Wilshire, B., "Obliteration of Latent Fingerprints," Journal of Forensic Sciences, Vol. 36, No. 5, Sept. 1991, pp. 1376-1386.

7. National Institute of Justice, "New Reagents for Development of Latent Fingerprints," National Institute of Justice Update, September 1995, PP???

8. Lincoln Land Community College, "Chemical Fingerprinting Materials," Available: http://www.llcc.cc.ll.us/justice/forensic/chemical.html

9. Edens, W., "Developing Fingerprints - Ninhydrin," Available: http://www.chicasaw.com/!waedens/fpc/nin.htm

10. Chester, B. K., Stone, J. M., and Rowe, W. F., "Use of the Omniprint ${ }^{\mathrm{TM}} 1000$ Alternate Light Source to Produce Fluorescence in Cyanoacrylate-Developed Latent Fingerprints Stained with Biological Stains and Commercial Fabric Dyes," Forensic Science International, Vol. 57, 1992, pp. 163-168.

11. Lincoln Land Community College, "SuperGlue," Available: http://www.llcc.cc.il.us/justice/forensic/superglue.html

12. Karlinszky, L., and Harkai, G., "Detection of Latent Fingerprints: Application of Cyanoacrylate for the Inside of Cars," Forensic Science International, Vol. 46, 1990, pp. 29-30.

13. German, E., "Cyanoacrylate (Superglue) Fuming Tips," Available: http://onin.com/fp/cyanoho.html

14. Bramble, S. K., "Fluorescence Spectroscopy as an Aid to Imaging Latent Fingermarks in the Ultraviolet," Journal of Forensic Sciences, Vol. 41, No. 6., 1996, pp. 1038-1041.

15. Lock, E. R. A.., Mazzella, W. D., and Margot, P., "A New Europium Chelate As a Fluorescent Dye for Cyanoacrylate Pretreated Fingerprints-EuTTAPhen: Europium ThenoylTrifluoroAcetone Ortho-Phenanthroline," Journal of Forensic Sciences, Vol. 40, No. 4., July 1995, pp. 654-658.

16. Dalyrymple, B. E., Duff, J. M., and Menzel, E. R., "Inherent Fingerprint Luminescence - Detection by Laser," Journal of Forensic Sciences," Vol. 22, 1997, pp. 106-115. 
17. Salares, V. R., Eves, C. R., and Carey, P. R., "On the Detection of Fingerprints by Laser Excited Luminescence, " Forensic Science International, Vol. 14, 1979, pp. 229-237.

18. Bramble, S. K., Creer, K. E., Gui Quiang, W., and Sheard, B., "Ultraviolet Luminescence from Latent Fingerprints," Forensic Science International, Vol. 59, 1993, pp. 3-14.

19. Yosef, N. B., Almog, J., Frank, A., Springer, E., and Canta, A. A., "Short UV Luminescence for Forensic Applications: Design of a Real-Time Observation System for Detection of Latent Fingerprints and Body Fluids," Journal of Forensic Sciences, Vol. 43, No. 2., 1998, pp. 299-304.

20. Menzel, E. R., "Latent Fingerprint Development with Lasers," ASTM Standardization News, March 1985, pp. 34-37.

21. Menzel, E. R., "Detection of Latent Fingerprints by Laser-Excited Luminescence," Analytical Chemistry, Vol. 61, No. 8, April 15, 1989, pp. 557A-561A.

22. Krauss, T. C., and Warlen, S. C., "The Forensic Science Use of Reflective Ultraviolet Photography, "Journal of Forensic Sciences, Vol. 30, No. 1, January 1985, pp. 262-268.

23. Keith, L. V., and Runion, W., "Short-wave UV Imaging Casework Applications," Journal of Forensic Identification, Vol. 48, No. 5, 1998, pp. 563-569.

24. Scenescope, Available: http://www.crimescope.com/Scene/scensco.htm

25. The KRIMESITE ${ }^{\mathrm{TM}}$ Scope Function, Available: http://www.ruvis.com/function.htm

26. Gabby, T., Winkleby, M. A., Boyce, W. T., Fisher, D. L., Lancaster, A., and Sensabaugh, G. F., "Sexual Abuse of Children. The Detection of Semen on Skin," American Joumal of Diseases of Children, Vol. 146, No. 6, June 1992, pp. 700-703. 


\section{DISTRIBUTION:}

3

Dr Richard Rau

National Institute of Justice

810 Seventh St N.W.

Washington, D.C. 20531

3

Dr Ned Godshall

Molecular Technologies Inc.

1105 Rocky Point NE

Albuquerque, NM 87123

3

Ann Talbot

Scientific Evidence Division Manager

Albuquerque Police Department

400 Roma NW

Albuquerque, NM 87102

3 MS0782 Smith, Gordon J., 5848

1 MS0859 Craft, David C., 2617

1 MS0986 Sandison, David R., 2663

1 MS0986 Vargo, Tim D., 2665

1 MS0987 Roehrig, Stephen C., 2664

1 MS0987 Ryerson, David E., 2664

5 MS0987 Smithpeter, Colin L.

1 MS9018 Central Technical Files, 8940-2

2 MS0899 Technical Library, 4916

1 MS0612 Review \& Approval Desk, 4912 for DOE/OSTI

1 MS0161 Patent and Licensing Office, 11500

1 MS1380 Technology Transfer, 4331 